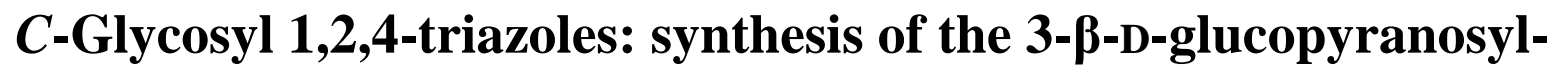

\section{1,5-disubstituted and 5- $\beta$-D-glucopyranosyl-1,3-disubstituted}

\section{variants}

Katalin E. Szabó, András Páhi, László Somsák*

Department of Organic Chemistry, University of Debrecen, PO Box 400, H-4002 Debrecen, Hungary

\begin{abstract}
Highly variable synthetic routes were elaborated toward trisubstituted $C$-glycopyranosyl 1,2,4-triazoles. $N$-Acyl-thioamide derivatives were obtained by acylation of $O$-perbenzoylated 2,6-anhydro-D-glycero-D-gulo-heptonothioamide by acid chlorides and of thioamides by $O$ perbenzoylated 2,6-anhydro-D-glycero-D-gulo-heptonoyl chloride. These precursors reacted with substituted hydrazines in a regioselective manner to yield 3- $\beta$-D-glucopyranosyl-1,5disubstituted- and 5- $\beta$-D-glucopyranosyl-1,3-disubstituted-1,2,4-triazoles, respectively. Analogous $N$-acyl-2,6-anhydro-heptonamides failed to give the above triazoles with hydrazines. $O$-Deprotection of the $C$-glucosyl 1,2,4-triazoles by the Zemplén method furnished test compounds which showed no inhibition against rabbit muscle glycogen phosphorylase $b$.
\end{abstract}

\section{Keywords}

C-Glycosyl heterocycle; 1,2,4-triazole; 2,6-anhydro-aldonamide; 2,6-anhydroaldonothioamide

\footnotetext{
${ }^{*}$ Corresponding author - Tel: +3652512900 ext 22348; Fax: +3652512744; E-mail:
} somsak.laszlo@science.unideb.hu 


\section{Introduction}

Although 1,2,4-triazoles have not been found as constituents of natural compounds, this heterocycle is frequently part of biologically active synthetic molecules, among them a wide range of marketed drugs and agricultural chemicals, and finds various applications in many other fields, e.g. in synthetic and analytical chemistry, uses as corrosion inhibitors, ligands of metal complexes, and functional materials. As a consequence of this broad utility and interest, a large variety of synthetic methods have been elaborated to get this heteroring and its derivatives resulting in miriads of 1,2,4-triazole containing compounds..$^{1-8}$

Carbohydrate derivatives of this heterocycle are much less available. Direct conjugation of the 1,2,4-triazole ring with sugars may occur by a $\mathrm{C}-\mathrm{N}$ or a $\mathrm{C}-\mathrm{C}$ bond. From the former class several examples of bioactive nucleoside analogues ${ }^{9}$ and $N^{1}{ }^{10-13}$ as well as $N^{4}$ glycopyranosides ${ }^{14}$ have been known. $C$-Glycosyl 1,2,4-triazoles are an even more uncommon type $\mathrm{e}^{15-18}$ and only in recent years has progress been made in this field with the syntheses of 3-glycopyranosyl-5-substituted-1,2,4-triazoles as glycogen phosphorylase inhibitors for potential antidiabetic use. ${ }^{19-24}$

As a continuation of our efforts in the above syntheses, the preparation of trisubstituted $C$ glycopyranosyl 1,2,4-triazoles was envisaged to generate molecules for structure-activity relationships of glycogen phosphorylase inhibitors and also for other potential biological applications. From the three possible isomeric structures (Scheme 1) some examples of the 3glycosyl-4,5-disubstituted-1,2,4-triazoles (I) were already described, ${ }^{21}$ therefore, this work has focused on the 3- $\beta$-D-glucopyranosyl-1,5-disubstituted (II) and 5- $\beta$-D-glucopyranosyl-1,3disubstituted (III) counterparts. 


\section{Results and Discussion}

A retrosynthetic analysis of the target compounds (Scheme 1) revealed two types of synthetic possibilities. Type A syntheses would require a 1,3-dipolar cycloaddition of nitriles with nitrilimines. Toward triazoles II route A would require a series of nitriles IV which themselves may also have to be prepared from other kinds of starting materials and $C$ glycosyl nitrilimine precursors with appropriate substituents like 5-glycosyl-2-substitutedtetrazoles $\mathbf{V}$ or hydrazonoyl halide derivatives of anhydro-aldonic acids VI which are also not readily available. Toward triazoles III route $\mathbf{A}$ would need well known glycosyl cyanides $\mathbf{X}$ and nitrilimine precursors such as 2,5-disubstituted-tetrazoles XI or hydrazonoyl halides XII. Actually, the latter type reaction $(\mathbf{X}+\mathbf{X I I})$ was studied to some extent, and a few 3- $\beta$-Dglycopyranosyl-1,5-disubstituted-1,2,4-triazoles were described. ${ }^{16,17}$ However, for getting a large series of compounds, multistep synthesis of each hydrazonoyl halide and/or 2,5disubstituted-tetrazole would be necessary. These requirements adumbrate rather labour intensive preparative work to get the precursors for both route A type syntheses. Therefore we turned to type $\mathbf{B}$ synthetic pathways which would need various hydrazines VII and acylation of easily available $C$-glycosyl formamides (anhydro-aldonamides) to get precursors VIII. In these cases the regioselectivity may be a challenge due to the possibly similar reactivity of the two electrophilic centres of VIII, therefore, the related acyl-thioamides IX and XIII were also taken into consideration. 
<smiles>[R]c1nnc([AlH2])n1[R]</smiles>

Synthesis realized by the transformation of $O$-peracylated 5-glycosyl tetrazoles with imidoyl chlorides. ${ }^{21}$

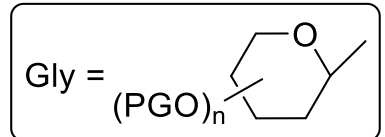

I

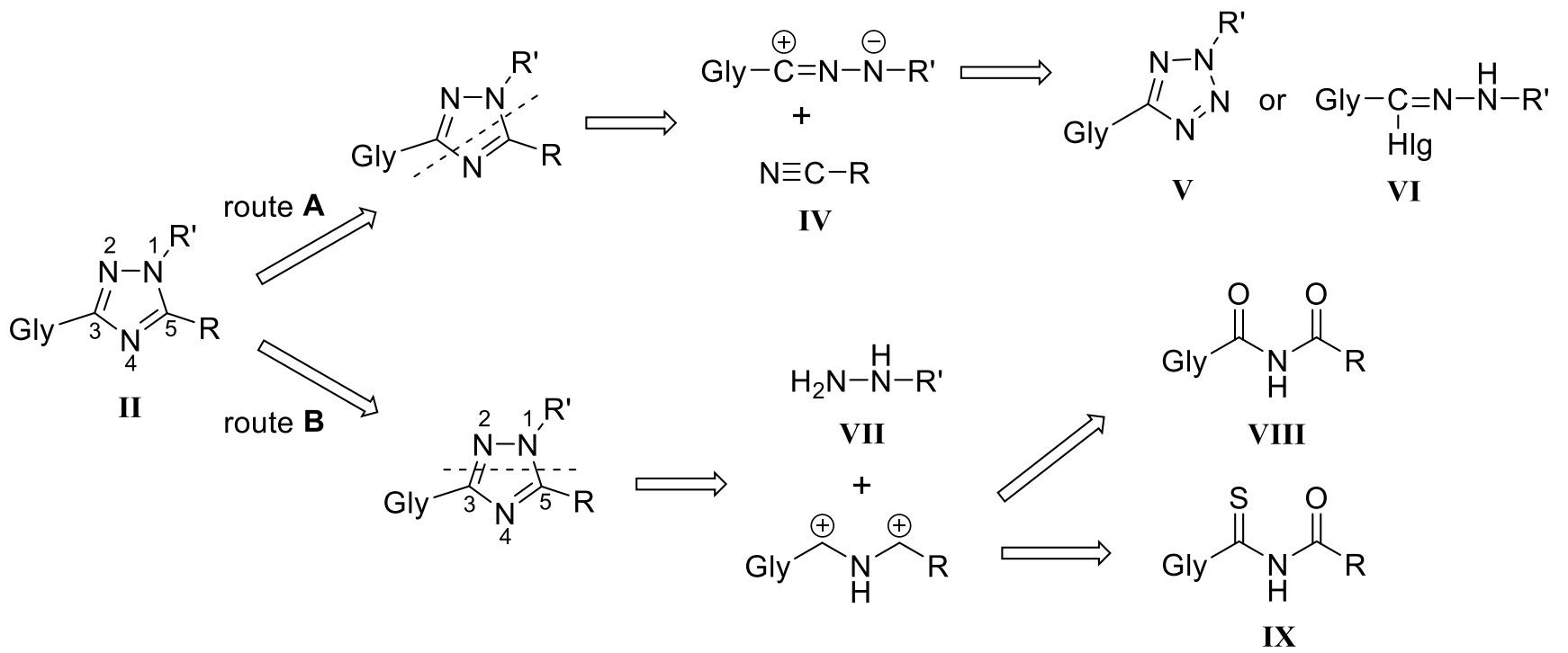

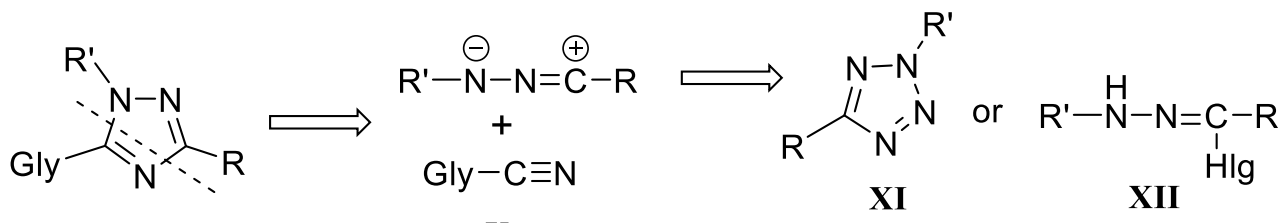

route $\mathrm{A}$

$\mathbf{X}$<smiles>[R]c1nc([AlH2])n([R1])n1</smiles>

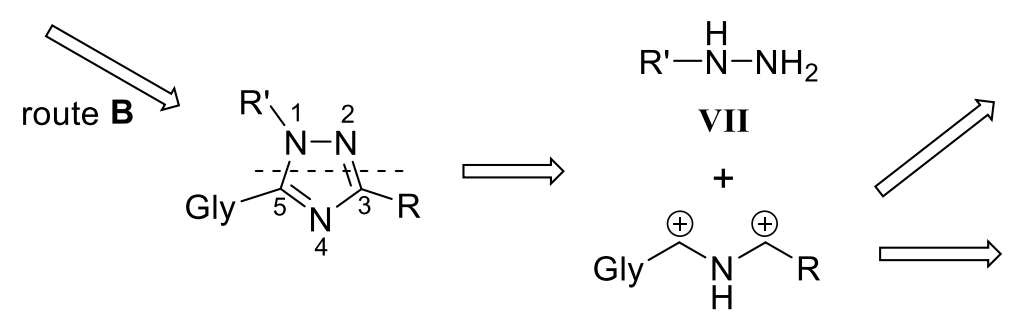<smiles></smiles>

VIII<smiles>[R]C(=S)NC(=O)C1CCCCC1</smiles>

XIII

Scheme 1. Isomers of trisubstituted $C$-glycopyranosyl 1,2,4-triazoles (I-III) and retrosynthetic analysis of the target compounds II and III. 
To get precursors of type VIII, the acylation of $O$-perbenzoylated 2,6-anhydro-heptonamide (C- $\beta$-D-glucopyranosyl formamide) $\mathbf{1}^{25}$ was studied (Scheme 1$)$. Under some conditions (1.515 equiv. of $\mathrm{Ac}_{2} \mathrm{O}$ in the presence of $\mathrm{N}$-bases, ${ }^{26,27}$ or $1-2$ equiv. of $\mathrm{AcCl}$ the presence of 1.2 equiv of $\mathrm{NaH}$ in THF, ${ }^{28,29} 2.5$ equiv. of $\mathrm{Bz}_{2} \mathrm{O}$ with catalytic $\mathrm{H}_{2} \mathrm{SO}_{4}$ in $\mathrm{CHCl}_{3}$ at reflux temperature ${ }^{30}$ ) incomplete conversion of the starting $\mathbf{1}$ was observed. Full transformation of $\mathbf{1}$ was achieved however, by using 5 equiv. of both an acid chloride and pyridine in $\mathrm{CHCl}_{3}$ at $\mathrm{r}$.

t. (Table 1), but the products $\mathbf{2 - 4}$ were accompanied by significant amounts of diacylated derivatives 5-7, respectively, and in the cases of aromatic acid chlorides, the dehydration ${ }^{31}$ of $\mathbf{1}$ to give glucosyl cyanide $\mathbf{8}^{25}$ (cf also Scheme 3) was the main reaction pathway.

Table 1. Experiments towards acylation of 2,6-anhydro-heptonamide 1

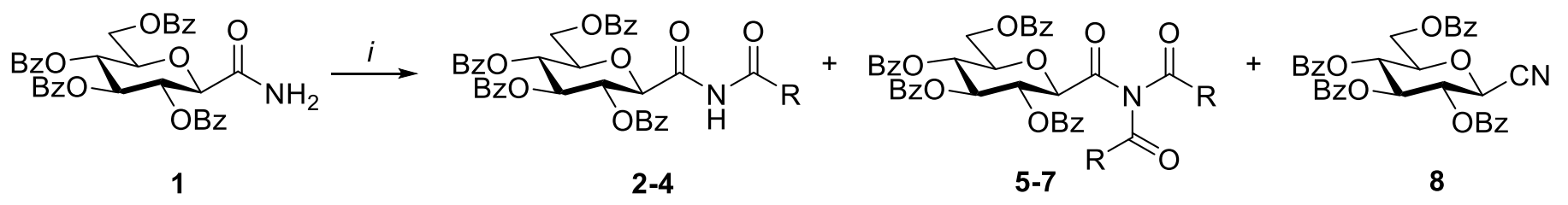

i) 5 equiv. $\mathrm{RCOCl}, 5$ equiv. pyridine, dry $\mathrm{CHCl}_{3}, \mathrm{rt}$

\begin{tabular}{lccc}
\hline $\mathrm{R}$ & & Yield (\%) & \\
\hline $\mathrm{Me}$ & $\mathbf{2}(51)$ & $\mathbf{5}(34)$ & - \\
$\mathrm{Ph}$ & $\mathbf{3}(26)$ & $\mathbf{6}(8)$ & $\mathbf{8}(67)$ \\
$1-$ Naphthyl & $\mathbf{4}(16)$ & $\mathbf{7}(15)$ & $\mathbf{8}(39)$ \\
\hline
\end{tabular}


An initial experiment towards the formation of a 1,2,4-triazole was carried out by reacting 2 with hydrazine hydrate or hydrazinium acetate. Surprisingly, the products of this transformation, obtained after column chromatography with an acetone-hexane eluent, proved to be amide $\mathbf{1}$ and acylhydrazone $\mathbf{1 0}$ (Scheme 2). This experiment proved the similar reactivity of the two $N$-carbonyls in $\mathbf{2}$ (the appearance of $\mathbf{1 0}$ can be explained by the formation of 9 which was condensed with acetone during the purification). Under the same conditions monoacyl-amides $\mathbf{3}$ and $\mathbf{4}$ gave only $\mathbf{1 0}$ as the final product in 74 and $72 \%$ yields, respectively. These observations indicated that $\mathrm{N}$-acyl-amides $\mathbf{2 - 4}$ could not serve as starting materials for the planned 1,2,4-triazole syntheses.

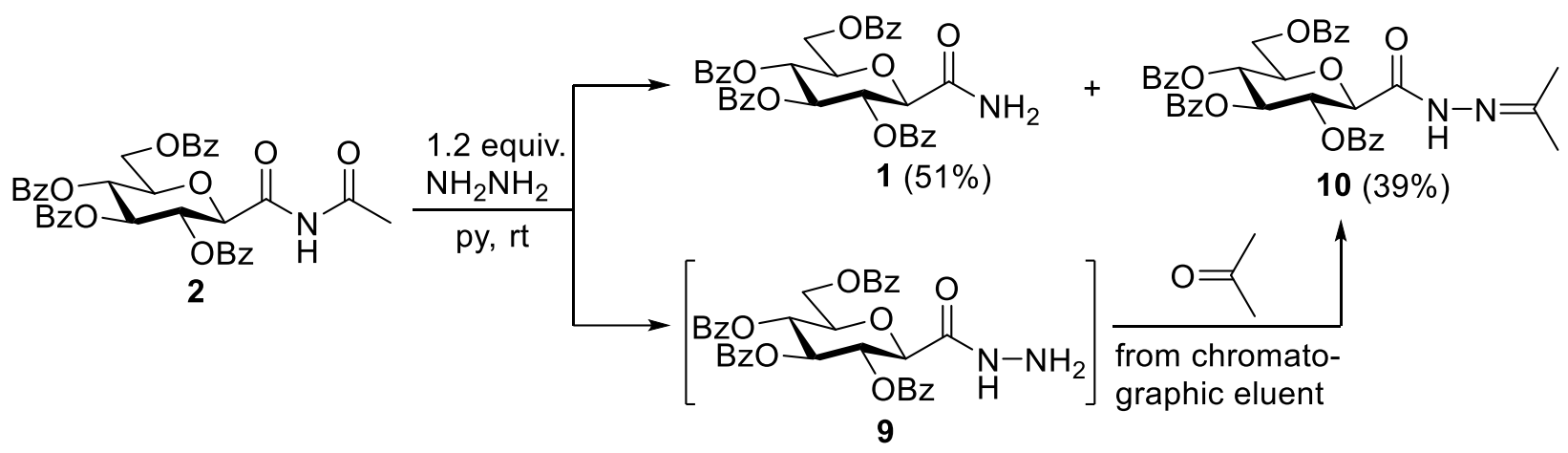

Scheme 2.

After this failure we turned to the preparation of precursors IX and XIII (Scheme 3). Towards IX, the $N$-acylation of 2,6-anhydro-aldonothioamide ( $C$ - $\beta$-D-glucopyranosyl thioformamide) $\mathbf{1 1}^{32}$ was smoothly carried out by several aliphatic acid chlorides to give excellent yields of 12-15. Unexpectedly, attempts to acylate 11 by aromatic acid chlorides ( $\mathrm{PhCOCl}, 1-$ naphthoylchloride) gave glucosyl cyanide $\mathbf{8}^{25}$ as the main product. Each reaction mixture contained a second product, probably the $N$-aroyl-thioamide, however, this could not be isolated in a pure state. This observation turned out to have a literature precedent, ${ }^{33}$ and thus, 
the formation of $\mathbf{8}$ as the primary product could be explained by an $S$-aroylation of the $\mathrm{CSNH}_{2}$ moiety to give $16(X=S)$ which then underwent a spontaneous loss of ArCOSH. Formation of $\mathbf{8}$ in $\mathrm{N}$-aroylations of $\mathbf{1}$ (Table 1) can be explained in an analogous manner via $\mathbf{1 6}(\mathrm{X}=\mathrm{O})$.

Precursors of type XIII were obtained by glycosylcarbonylation of thioacetamide and thiobenzamide with 2,6-anhydro-aldonoyl chloride ( $C$ - $\beta$-D-glucopyranosylformyl chloride) $\mathbf{1 7 ^ { 2 0 , 3 4 }}$ and the expected $\mathbf{1 8}$ and $\mathbf{1 9}$, respectively, could be isolated in acceptable yields (Scheme 3).

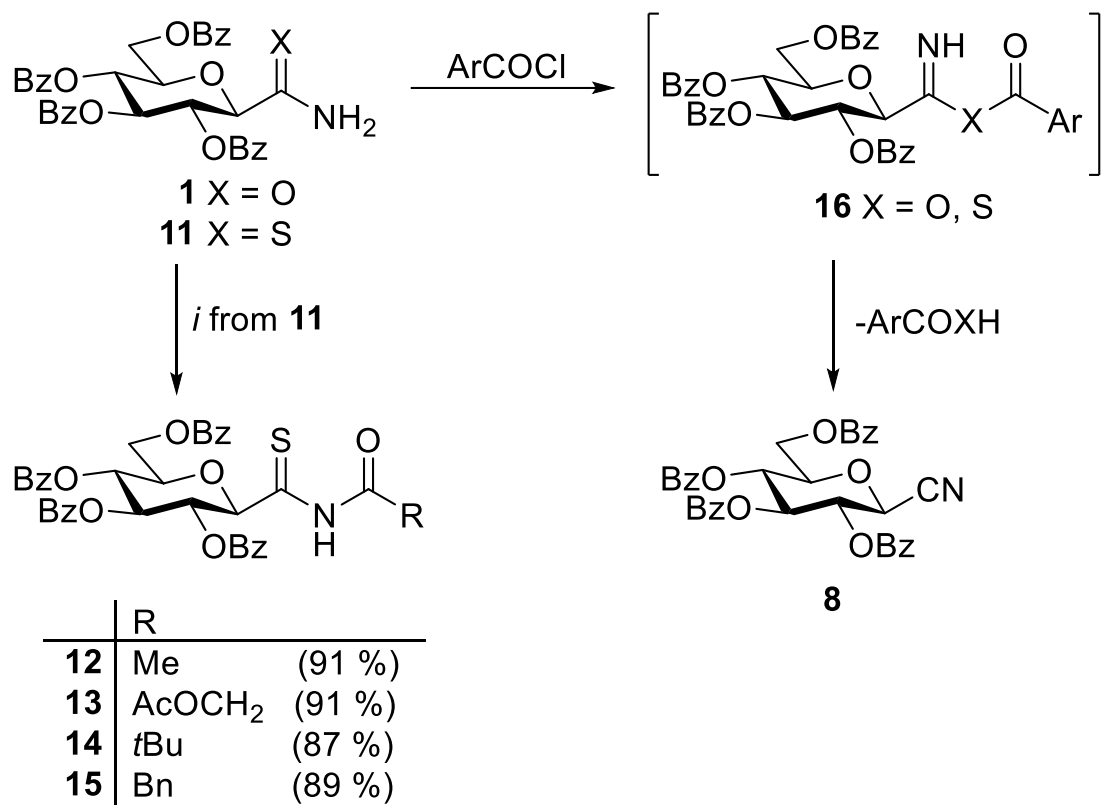

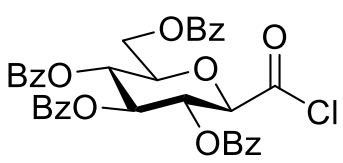

17
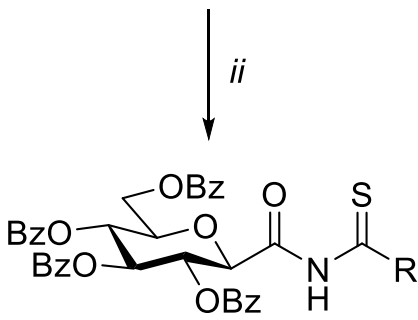

\begin{tabular}{l|ll} 
& $\mathrm{R}$ & \\
\hline 18 & $\mathrm{Me}$ & $(58 \%)$ \\
19 & $\mathrm{Ph}$ & $(71 \%)$
\end{tabular}

Scheme 3. Reagents and conditions: i) 1.5 equiv. $\mathrm{RCOCl}, 1.5$ equiv. pyridine, dry $\mathrm{CHCl}_{3}$, rt; ii) 2 equiv. $\mathrm{RCSNH}_{2}, 2$ equiv. pyridine, dry $\mathrm{CH}_{3} \mathrm{CN}$, rt.

With the $N$-acyl-thioamide precursors in hand, the syntheses of the target 1,2,4-triazoles were carried out. Compounds 12-15 were reacted with hydrazinium acetate in pyridine at r. t.

(Table 2) to give 20, 24, 27 and $\mathbf{3 0}$, respectively, from which the first three were identical, when compared, with previously prepared compounds, ${ }^{20}$ thereby proving the suitability of these precursors for the triazole synthesis. Reactions of 12-15 with substituted hydrazines 
(phenylhydrazine, 2-hydroxyethyl-hydrazine and tosylhydrazine) gave the corresponding 2123, 25, 26, 28, 29, 31, and 32, respectively, in good yields. $O$-Debenzoylation of 21, 22, 24, 25, 27-31 by the Zemplén method gave 3- $\beta$-D-glucopyranosyl-1,5-disubstituted-1,2,4triazoles 33-41, respectively, in good yields.

Reactions of $N$-acyl-thioamides 18 and 19 with hydrazinium acetate gave the known 3- $\beta$-Dglucopyranosyl-5-substituted-1,2,4-triazoles ${ }^{20} \mathbf{2 0}$ and 43, respectively (Table 3). With substituted hydrazines the expected 1,2,4-triazoles 42, 44-47 were obtained in varying yields, which were $O$-deprotected by the Zemplén protocol to give 5- $\beta$-D-glucopyranosyl-1,3disubstituted-1,2,4-triazoles 48-52, respectively, in excellent yields. When 19 was transformed with tosylhydrazine, triazole $\mathbf{4 3}$ was isolated indicating spontaneous loss of the tosyl moiety under the applied conditions similarly to the observations made in reactions of $N^{1}$-tosyl- $C$-(2,3,4,6-tetra- $O$-benzoyl- $\beta$-D-glucopyranosyl)formamidrazone and acid chlorides. $^{20}$ 
Table 2. Synthesis of 3- $\beta$-D-glucopyranosyl-1,5-disubstituted-1,2,4-triazoles

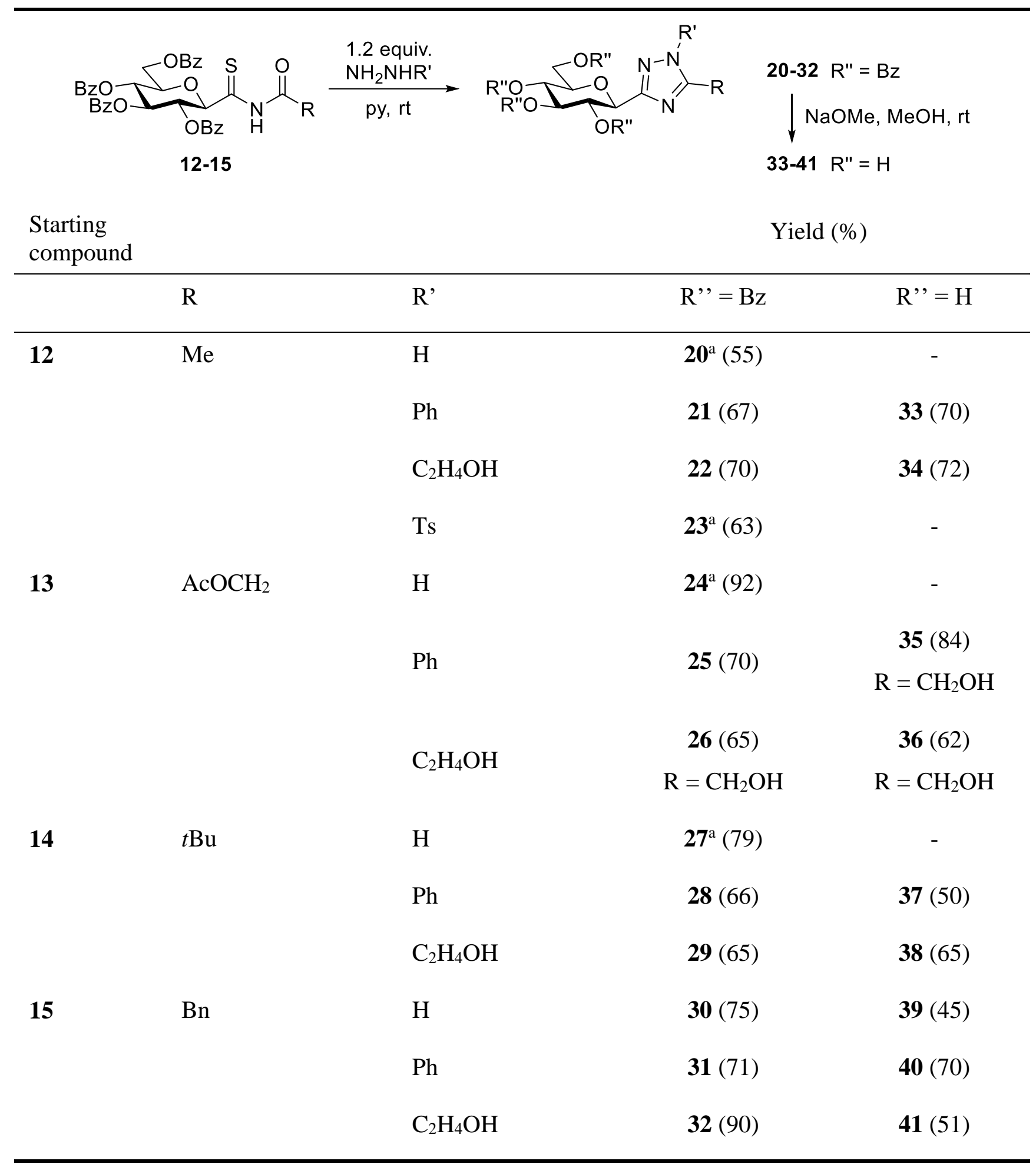

\footnotetext{
a The compound was identical with the one obtained from $N^{1}$-tosyl- $C$-(2,3,4,6-tetra- $O$-benzoyl- $\beta$-Dglucopyranosyl)formamidrazone and the corresponding acid chloride. ${ }^{20}$
} 
Table 3. Synthesis of 5- $\beta$-D-glucopyranosyl-1,3-disubstituted-1,2,4-triazoles

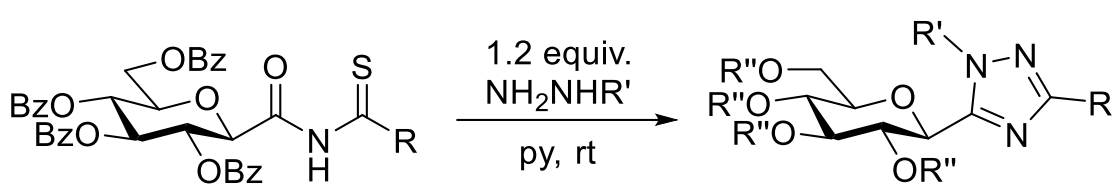

20, $42-47 R^{\prime \prime}=B z$

18,19

48-52 $\mathrm{R}^{\prime \prime}=\mathrm{H}$

Starting
compound

Yield $(\%)$

\begin{tabular}{|c|c|c|c|c|}
\hline & $\mathrm{R}$ & $\mathrm{R}^{\prime}$ & $\mathrm{R}^{\prime \prime}=\mathrm{Bz}$ & $\mathrm{R}^{\prime \prime}=\mathrm{H}$ \\
\hline \multirow[t]{2}{*}{18} & $\mathrm{Me}$ & $\mathrm{H}$ & $20^{a}(57)$ & - \\
\hline & & $\mathrm{Ph}$ & $42(65)$ & $48(96)$ \\
\hline \multirow[t]{6}{*}{19} & $\mathrm{Ph}$ & $\mathrm{H}$ & $\mathbf{4 3}^{\mathrm{a}}(35)$ & - \\
\hline & & $\mathrm{Ph}$ & $44(90)$ & $49(83)$ \\
\hline & & $\mathrm{C}_{2} \mathrm{H}_{4} \mathrm{OH}$ & $45(90)$ & $\mathbf{5 0}(98)$ \\
\hline & & $t \mathrm{Bu}$ & $46(60)$ & $51(99)$ \\
\hline & & $3-\mathrm{Cl}-\mathrm{C}_{6} \mathrm{H}_{4}$ & $47(72)$ & $52(99)$ \\
\hline & & Ts (in the hydrazine) & $\begin{array}{l}\mathbf{4 3}^{\mathrm{a}}(62) \\
\left(\mathrm{R}^{\prime}=\mathrm{H}\right)\end{array}$ & - \\
\hline
\end{tabular}

a The compound was identical with the one obtained from $N^{1}$-tosyl- $C$-(2,3,4,6-tetra- $O$-benzoyl- $\beta$-Dglucopyranosyl)formamidrazone and the corresponding acid chloride. ${ }^{20}$

The different reactivity of the $\mathrm{C}=\mathrm{O}$ and $\mathrm{C}=\mathrm{S}$ moieties in the $N$-acyl-thioamides leaves not much doubt about the regioselectivity of the above ring closing reactions, i. e. the more nucleophilic terminal nitrogen of the substituted hydrazine reagent reacts with the more electrophilic $\mathrm{C}=\mathrm{S}$ group, it was found appropriate to prove this at least in one case. Thus, nuclear Overhauser effects (NOE) were measured in isomeric triazoles $\mathbf{3 3}$ and $\mathbf{4 8}$ (Figure 1) 
to show the vicinity of the $\mathrm{Me}$ and $\mathrm{Ph}$ groups in the former and that of the $\mathrm{H}-1$ and $\mathrm{Ph}$ in the latter.
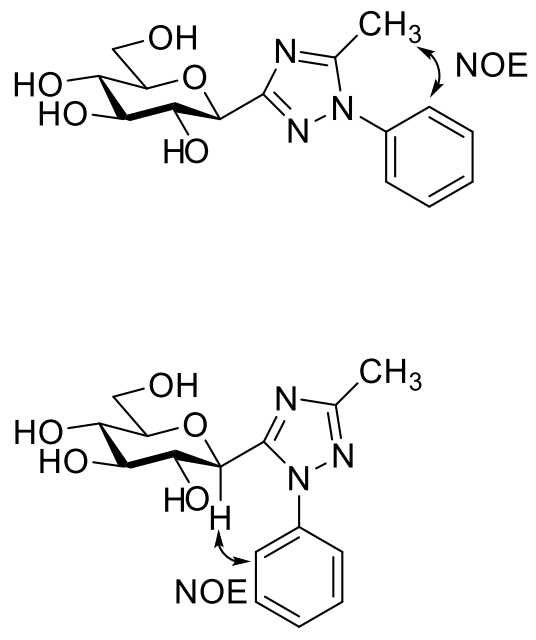

Figure 1. Evidence of the isomeric structures for 1,2,4-triazoles 33 and $\mathbf{4 8}$

The unprotected compounds 33-41 and 48-52 were assayed against rabbit muscle glycogen phosphorylase $b(\mathrm{RMGP} b)$ as described before,${ }^{35}$ however, none of them showed inhibition in $625 \mu \mathrm{M}$ concentration. In our previous studies ${ }^{19,21}$ several 3-glucopyranosyl-5-substituted1,2,4-triazoles were found active against RMGP $b$ in the submicromolar range. The good inhibitory effect of those compounds was attributed to the hydrogen bond donor ability of the heterocycle, a feature discussed in a recent review. ${ }^{18}$ In the context of structure-activity relationships of GP inhibitors, the present results underline the importance of the H-bridge formation since this possibility is absent in the trisubtituted 1,2,4-triazoles. Furthermore, the additional substituent of the triazole ring may also impede binding of these molecules to the active site of the enzyme. 
In conclusion, reactions of substituted hydrazines and sugar derived $N$-acyl-thioamide type precursors facilitate versatile syntheses of 3- $\beta$-D-glucopyranosyl-1,5-disubstituted- and 5- $\beta$-Dglucopyranosyl-1,3-disubstituted-1,2,4-triazoles. The only limitation of the method is that in

the 3- $\beta$-D-glucopyranosyl-1,5-disubstituted isomers no aromatic substituent can be introduced in the 5-position due to facile desulfuration of the corresponding 2,6-anhydroaldonothioamide by aromatic acid chlorides.

\section{Experimental}

\section{General Methods}

Melting points were measured on a Kofler hot-stage and are uncorrected. Optical rotations were determined with a Perkin-Elmer 241 polarimeter at rt. NMR spectra were recorded with Bruker $360\left(360 / 90 \mathrm{MHz}\right.$ for $\left.{ }^{1} \mathrm{H} /{ }^{13} \mathrm{C}\right)$ or Bruker $400\left(400 / 100 \mathrm{MHz}\right.$ for $\left.{ }^{1} \mathrm{H} /{ }^{13} \mathrm{C}\right)$ spectrometers. Chemical shifts are referenced to the internal TMS $\left({ }^{1} \mathrm{H}\right)$, or to the residual solvent signals $\left({ }^{13} \mathrm{C}\right)$. Mass spectra were obtained by a Thermo Scientific LTQ XL instrument or by a Bruker micrOTOF-Q instrument. TLC was performed on DC-Alurolle Kieselgel 60 F 254 (Merck), and the plates were visualised under UV light and by gentle heating (generally no spray reagent was used but, if more intense charring was necessary, the plate was sprayed with the following solution: abs. EtOH (95 mL), $\mathrm{ccH}_{2} \mathrm{SO}_{4}(5 \mathrm{~mL})$ anisaldehyde $\left.(1 \mathrm{~mL})\right)$. For column chromatography Kieselgel 60 (Merck, particle size 0.063-0.200 mm) was used. MeCN and $\mathrm{CHCl}_{3}$ were distilled from $\mathrm{P}_{4} \mathrm{O}_{10}$ and stored over $4 \AA$ molecular sieves. Pyridine was distilled from $\mathrm{KOH}$ and stored over $\mathrm{KOH}$ pellets. $\mathrm{MeOH}$ was purified by distillation after refluxing for a couple of hours with magnesium turnings and iodine. Organic solutions were dried over anhydrous $\mathrm{MgSO}_{4}$ and concentrated under diminished pressure at $40{ }^{\circ} \mathrm{C}$ (water bath). Acid chlorides, thioamides, hydrazine hydrate and hydrazinium acetate, substituted hydrazines were purchased from Sigma-Aldrich. $C$-(2,3,4,6-tetra- $O$-benzoyl- $\beta$-D- 
glucopyranosyl)formamide ${ }^{25}(\mathbf{1})$ and $C$-(2,3,4,6-tetra- $O$-benzoyl- $\beta$-D-

glucopyranosyl)thioformamide ${ }^{32}(\mathbf{1 1})$ were synthesized according to published procedures.

\section{General procedure I for the acylation of $C$-(2,3,4,6-tetra- $O$-benzoyl- $\beta$-D-} glucopyranosyl)formamide.

To a solution of $C$-(2,3,4,6-tetra- $O$-benzoyl- $\beta$-D-glucopyranosyl)formamide ${ }^{25}(\mathbf{1})$ in anhydrous $\mathrm{CHCl}_{3}(10 \mathrm{~mL} / \mathrm{mmol})$ the corresponding acid chloride (5.0 equiv.) and pyridine (5.0 equiv.) were added. The reaction mixture was stirred at $\mathrm{rt}$ and monitored by TLC (1:8 EtOAc/toluene). After complete conversion of $\mathbf{1}$ the mixture was diluted with $\mathrm{CHCl}_{3}(30 \mathrm{~mL})$ and extracted with $\mathrm{NaHCO}_{3}(2 \times 10 \mathrm{~mL})$ then with water $(1 \times 10 \mathrm{~mL})$. The organic phase was dried over $\mathrm{MgSO}_{4}$, concentrated under diminished pressure, and the crude product was purified by column chromatography.

General procedure II for the preparation of $N$-acyl- $C$-(2,3,4,6-tetra- $O$-benzoyl- $\beta$-Dglucopyranosyl)thioformamides.

To a solution of the $C$-(2,3,4,6-tetra- $O$-benzoyl- $\beta$-D-glucopyranosyl)thioformamide ${ }^{32}(\mathbf{1 1})$ in anhydrous $\mathrm{CHCl}_{3}(10 \mathrm{~mL} / \mathrm{mmol})$ the corresponding acid chloride (1.5 equiv.) and pyridine (1.5 equiv.) were added. The reaction mixture was stirred at $\mathrm{rt}$ and monitored by TLC $(1: 8$ EtOAc/toluene). After complete conversion of $\mathbf{1 1}$ the mixture was diluted with $\mathrm{CHCl}_{3}(30$ $\mathrm{mL})$ and extracted with $\mathrm{NaHCO}_{3}(2 \times 10 \mathrm{~mL})$ then with water $(1 \times 10 \mathrm{~mL})$. The organic phase was dried over $\mathrm{MgSO}_{4}$, concentrated under diminished pressure, and the crude product was purified by column chromatography. 


\section{General procedure III for the preparation of $N$-(2,3,4,6-tetra- $O$-benzoyl- $\beta$-D-}

glucopyranosylcarbonyl) thioamides.

$C$-(2,3,4,6-tetra- $O$-benzoyl- $\beta$-D-glucopyranosyl)formic acid was boiled in $\mathrm{SOCl}_{2}(10$ $\mathrm{mL} / \mathrm{mmol}$ ) for 2 hours, then the excess of $\mathrm{SOCl}_{2}$ was evaporated under reduced pressure. Traces of the reagents were removed by repeated co-evaporations with anhydorus toluene. The resulting acid chloride $17^{20}$ was dissolved in anhydrous $\mathrm{CH}_{3} \mathrm{CN}(15 \mathrm{~mL} / \mathrm{mmol})$, and the solution of the corresponding thioamide (2.0 equiv.) and pyridine (2.0 equiv.) in anhydrous $\mathrm{CH}_{3} \mathrm{CN}(15 \mathrm{~mL} / \mathrm{mmol})$ were added dropwise over 20 minutes. The reaction mixture was stirred at rt. After complete conversion of $\mathbf{1 7}$ (monitored by TLC, 1:1 EtOAc/hexane, $12 \mathrm{~h}$ ) the solvent was removed and the residue was purified by column chromatography.

\section{General procedure IV for the preparation of 1,5-disubstituted-3-(2',3',4',6'-tetra- $O$ - benzoyl- $\beta$-D-glucopyranosyl)-1,2,4-triazoles and 1,3-disubstituted-5-(2',3',4',6'-tetra- $O$ - benzoyl- $\beta$-D-glucopyranosyl)-1,2,4-triazoles.}

To a solution of an $N$-acyl-thioamide $\mathbf{1 2 - 1 5}, \mathbf{1 8}$, or 19 in anhydrous pyridine $(10 \mathrm{~mL} / \mathrm{mmol})$ the corresponding hydrazine (1.2 equiv.) was added, and the reaction mixture was stirred at $\mathrm{rt}$ for $2 \mathrm{~h}$. After complete conversion of the starting material (monitored by TLC, 2:1 EtOAc/hexane) the solvent was removed and the residue was purified by column chromatography.

\section{General procedure $V$ for the removal of $O$-acyl protecting groups by the Zemplén} protocol.

An $O$-acylated compound was dissolved in dry $\mathrm{MeOH}(5 \mathrm{~mL} / 100 \mathrm{mg})$ and 1-2 drops of a 1 $\mathrm{M}$ methanolic $\mathrm{NaOMe}$ solution were added. The mixture was kept at $\mathrm{rt}$ and monitored by TLC (3:7 $\left.\mathrm{MeOH} / \mathrm{CHCl}_{3}\right)$. When the starting material was consumed the mixture was 
neutralised with a cation exchange resin Amberlyst $15\left(\mathrm{H}^{+}\right.$form $)$or with acetic acid, then the resin was filtered off and the solvent removed. The residue was purified by column chromatography.

\section{Synthesis and characterization of the compounds}

\section{$N$-Acetyl- $C$-(2,3,4,6-tetra- $O$-benzoyl- $\beta$-D-glucopyranosyl)formamide (2)}

Prepared from compound $1(0.25 \mathrm{~g}, 0.40 \mathrm{mmol})$ and acetyl chloride $(0.14 \mathrm{~mL}, 2.00 \mathrm{mmol})$ according to general procedure I (reaction time: $12 \mathrm{~h}$ ). Purified by column chromatography (1:4 EtOAc/hexane) to give $0.14 \mathrm{~g}(51 \%)$ white amorphous solid. $\mathrm{R}_{\mathrm{f}}=0.27(1: 2$ EtOAc/hexane); $[\alpha]_{\mathrm{D}}-2\left(\mathrm{c} 0.58, \mathrm{CHCl}_{3}\right) ;{ }^{1} \mathrm{H} \mathrm{NMR}\left(400 \mathrm{MHz}, \mathrm{CDCl}_{3}\right) \delta(\mathrm{ppm}): 8.99(1 \mathrm{H}, \mathrm{s}$, $\mathrm{NH}), 8.06(2 \mathrm{H}, \mathrm{dd}, J=8.2,1.1 \mathrm{~Hz}$, aromatics), 7.97-7.91 (4H, m, aromatics), 7.83 (2H, dd, $J$ $=8.2,1.1 \mathrm{~Hz}$, aromatics), 7.58-7.25 (12H, m, aromatics), $5.99(1 \mathrm{H}$, pseudo $\mathrm{t}, J=9.2 \mathrm{~Hz}, \mathrm{H}-2$ ' or H-3' or H-4'), 5.75-5.66 (2H, m, H-2' and/or H-3' and/or H-4'), 4.72 (1H, dd, $J=12.5,2.6$ Hz, H-6'a ), 4.55 (1H, dd, $J=12.5,5.3$ Hz, H-6'b), 4.37 (1H, d, $J=9.5$ Hz, H-1'), 4.27 (1H, ddd, $J=9.6,5.2,2.7 \mathrm{~Hz}, \mathrm{H}-5$ ') $2.37\left(3 \mathrm{H}, \mathrm{s}, \mathrm{CH}_{3}\right) ;{ }^{13} \mathrm{C} \mathrm{NMR}\left(100 \mathrm{MHz}, \mathrm{CDCl}_{3}\right) \delta(\mathrm{ppm})$ : 171.9, 166.4, 166.0, 165.7, 165.4, $165.2(\mathrm{C}=\mathrm{O}), 133.7-128.5$ (aromatics), 76.8, 76.5, 73.2, 69.8, $68.9\left(\mathrm{C}-1^{\prime}-\mathrm{C}^{-5}\right.$ ') $, 62.8\left(\mathrm{C}-6^{\prime}\right), 25.4\left(\mathrm{CH}_{3}\right)$. ESI-MS positive mode $(\mathrm{m} / \mathrm{z}):[\mathrm{M}+\mathrm{Na}]^{+}$, found 688.18; $\mathrm{C}_{37} \mathrm{H}_{31} \mathrm{NNaO}_{11}$ requires 688.180. Anal. Calcd for $\mathrm{C}_{37} \mathrm{H}_{31} \mathrm{NO}_{11}$ : C, 66.76; $\mathrm{H}$, 4.69; N, 2.10. Found: C, 66.88; H, 4.75; N, 2.08.

\section{$N$-Benzoyl- $C$-(2,3,4,6-tetra- $O$-benzoyl- $\beta$-D-glucopyranosyl)formamide (3)}

Prepared from compound 1 ( $0.25 \mathrm{~g}, 0.40 \mathrm{mmol})$ and benzoyl chloride $(0.23 \mathrm{~mL}, 2.00 \mathrm{mmol})$ according to general procedure I (reaction time: $3 \mathrm{~d}$ ). Purified by column chromatography (1:3 EtOAc/hexane) to give $0.08 \mathrm{~g}(26 \%)$ white amorphous solid. $\mathbf{R}_{\mathrm{f}}=0.38(1: 1$ 
EtOAc/hexane); $[\alpha]_{\mathrm{D}}-1$ (c $\left.0.51, \mathrm{CHCl}_{3}\right) ;{ }^{1} \mathrm{H} \mathrm{NMR}\left(400 \mathrm{MHz}, \mathrm{CDCl}_{3}\right) \delta(\mathrm{ppm}): 9.47(1 \mathrm{H}, \mathrm{s}$, $\mathrm{NH}), 8.01(2 \mathrm{H}, \mathrm{dd}, J=8.2,1.1 \mathrm{~Hz}$, aromatics), 7.96-7.93 (3H, m, aromatics), 7.83 (3H, d, $J=$ 8.1 Hz, aromatics), 7.56-7.26 (17H, m, aromatics), $6.00(1 \mathrm{H}$, pseudo t, $J=9.2 \mathrm{~Hz}, \mathrm{H}-2$ ' or $\mathrm{H}-$ 3' or H-4'), 5.84 (1H, pseudo t, $J=9.4 \mathrm{~Hz}, \mathrm{H}-2$ ' or $\mathrm{H}-3$ ' or $\mathrm{H}-4$ '), 5.76 (1H, pseudo t, $J=9.5$ Hz, H-2' or H-3' or H-4'), 4.78 (1H, dd, $J=12.5,2.5$ Hz, H-6'a ), 4.69 (1H, d, $J=9.5$ Hz, H$\left.1^{\prime}\right), 4.58\left(1 \mathrm{H}, \mathrm{dd}, J=12.5,5.3 \mathrm{~Hz}, \mathrm{H}-6\right.$ 'b), $4.32\left(1 \mathrm{H}, \mathrm{ddd}, J=10.0,5.3,2.5 \mathrm{~Hz}, \mathrm{H}-5{ }^{\prime}\right) ;{ }^{13} \mathrm{C}$ NMR $\left(100 \mathrm{MHz}, \mathrm{CDCl}_{3}\right) \delta(\mathrm{ppm}): 166.4,165.8,165.5,165.3,165.3,164.7(\mathrm{C}=\mathrm{O}), 133.8-$ 128.0 (aromatics), 76.9, 76.7, 73.6, 69.5, 68.9 (C-1'-C-5'), 62.5 (C-6'). ESI-MS positive mode $(\mathrm{m} / \mathrm{z})$ : calcd for $\mathrm{C}_{42} \mathrm{H}_{33} \mathrm{NNaO}_{11}\left([\mathrm{M}+\mathrm{Na}]^{+}\right)$: 750.195. Found: 750.19. Anal. Calcd for $\mathrm{C}_{42} \mathrm{H}_{33} \mathrm{NO}_{11}$ : C, 69.32; H, 4.57; N, 1.92. Found: C, 69.46; H, 4.47; N, 1.90 .

\section{$N$-(1-Naphthoyl)-C-(2,3,4,6-tetra- $O$-benzoyl- $\beta$-D-glucopyranosyl)formamide (4)}

Prepared from compound $1(0.20 \mathrm{~g}, 0.32 \mathrm{mmol})$ and naphthoyl chloride $(0.24 \mathrm{~mL}, 1.6 \mathrm{mmol})$ according to general procedure I (reaction time: $1 \mathrm{~d}$ ). Purified by column chromatography (1:1:3 EtOAc/toluene/hexane) to give $0.04 \mathrm{~g}(16 \%)$ white amorphous solid. $\mathrm{R}_{\mathrm{f}}=0.42(1: 3: 1$ EtOAc/toluene/hexane); $[\alpha]_{\mathrm{D}}-4\left(\mathrm{c} 0.53, \mathrm{CHCl}_{3}\right) ;{ }^{1} \mathrm{H} \mathrm{NMR}\left(400 \mathrm{MHz}, \mathrm{CDCl}_{3}\right) \delta$ (ppm): 9.29 (1H, s, NH), $8.33(1 \mathrm{H}, \mathrm{d}, J=7.3 \mathrm{~Hz}$, aromatics), 7.98-7.79 (9H, m, aromatics), $7.72(1 \mathrm{H}, \mathrm{d}, J$ $=6.8 \mathrm{~Hz}$, aromatics $), 7.57-7.20(16 \mathrm{H}, \mathrm{m}$, aromatics $), 5.98\left(1 \mathrm{H}\right.$, pseudo $\mathrm{t}, J=9.2 \mathrm{~Hz}, \mathrm{H}-2^{\prime}$ or H-3' or H-4'), 5.84 (1H, pseudo t, $J=9.4 \mathrm{~Hz}, \mathrm{H}-2^{\prime}$ or $\mathrm{H}-3^{\prime}$ or $\mathrm{H}-4$ '), 5.71 (1H, pseudo t, $J=$ 9.5 Hz, H-2' or H-3' or H-4'), 4.67 (2H, m, H-6'and H-1'), 4.52 (1H, dd, J = 12.4, 5.4 Hz, H6’b), $4.28\left(1 \mathrm{H}, \mathrm{ddd}, J=10.1,5.5,2.6 \mathrm{~Hz}, \mathrm{H}-5\right.$ '); ${ }^{13} \mathrm{C} \mathrm{NMR}\left(90 \mathrm{MHz}, \mathrm{CDCl}_{3}\right) \delta(\mathrm{ppm}): 166.8$, 166.3, 165.8, 165.5, 165.3, 165.3 (C=O), 133.8-124.6 (aromatics), 76.9, 76.6, 73.6, 69.5, 68.9 (C-1'-C-5'), 62.6 (C-6'). ESI-MS positive mode (m/z): calcd for $\mathrm{C}_{46} \mathrm{H}_{35} \mathrm{NNaO}_{11}\left([\mathrm{M}+\mathrm{Na}]^{+}\right)$: 800.211. Found: 800.21. Anal. Calcd for $\mathrm{C}_{46} \mathrm{H}_{35} \mathrm{NO}_{11}$ : C, 71.04; H, 4.54; N, 1.80. Found: C, 71.28; H, 4.60; N, 1.82 . 


\section{$N, N$-Diacetyl- $C$-(2,3,4,6-tetra- $O$-benzoyl- $\beta$-D-glucopyranosyl)formamide (5)}

Prepared from compound $1(0.25 \mathrm{~g}, 0.40 \mathrm{mmol})$ and acetyl chloride $(0.14 \mathrm{~mL}, 2.00 \mathrm{mmol})$ according to general procedure I (reaction time: $12 \mathrm{~h}$ ). Purified by column chromatography (1:4 EtOAc/hexane) to give $0.10 \mathrm{~g}(34 \%)$ white amorphous solid. $\mathrm{R}_{\mathrm{f}}=0.33(1: 2$ EtOAc/hexane); $[\alpha]_{\mathrm{D}}+80\left(\mathrm{c} 0.51, \mathrm{CHCl}_{3}\right) ;{ }^{1} \mathrm{H} \mathrm{NMR}\left(400 \mathrm{MHz}, \mathrm{CDCl}_{3}\right) \delta(\mathrm{ppm}): 8.09(2 \mathrm{H}, \mathrm{d}$, $J=8.3 \mathrm{~Hz}$, aromatics), 7.96-7.90 (4H, m, aromatics), $7.84(2 \mathrm{H}, \mathrm{d}, J=8.4 \mathrm{~Hz}$, aromatics), 7.60-7.25 (12H, m, aromatics), 6.00 (1H, pseudo t, $J=9.5 \mathrm{~Hz}, \mathrm{H}-2$ ' or H-3' or H-4'), 5.92 (1H, pseudo t, $J=9.3 \mathrm{~Hz}, \mathrm{H}-2^{\prime}$ or H-3' or H-4'), 5.69 (1H, pseudo t, $J=9.6 \mathrm{~Hz}, \mathrm{H}-2$ ' or H-3' or H-4'), 4.84 (1H, d, $J=9.6 \mathrm{~Hz}, \mathrm{H}-1$ '), 4.67 (1H, dd, $J=12.6,2.4 \mathrm{~Hz}, \mathrm{H}-6$ 'a ), 4.42 (1H, dd, $J=12.6,5.1 \mathrm{~Hz}, \mathrm{H}-6$ 'b), $4.16\left(1 \mathrm{H}, \mathrm{ddd}, J=9.6,5.1,2.4 \mathrm{~Hz}, \mathrm{H}-5\right.$ '), $2.33\left(6 \mathrm{H}, \mathrm{s}, 2 \times \mathrm{CH}_{3}\right) ;{ }^{13} \mathrm{C}$ NMR $\left(90 \mathrm{MHz}, \mathrm{CDCl}_{3}\right) \delta(\mathrm{ppm}): 174.2,174.2,170.5,166.1,165.9,165.2,165.1(\mathrm{C}=\mathrm{O})$, 133.7-128.4 (aromatics), 77.1, 76.4, 74.2, 69.2, 68.7 (C-1'-C-5'), 62.7 (C-6'), 26.3 (2 × $\left.\mathrm{CH}_{3}\right)$. ESI-MS positive mode $(\mathrm{m} / \mathrm{z})$ : calcd for $\mathrm{C}_{39} \mathrm{H}_{33} \mathrm{NNaO}_{12}\left([\mathrm{M}+\mathrm{Na}]^{+}\right)$: 730.190 . Found: 730.19. Anal. Calcd for $\mathrm{C}_{39} \mathrm{H}_{33} \mathrm{NO}_{12}$ : C, 66.19; H, 4.70; N, 1.98. Found: $\mathrm{C}, 66.32 ; \mathrm{H}, 4.76 ; \mathrm{N}$, 1.93.

\section{$N, N$-Dibenzoyl- $C$-(2,3,4,6-tetra- $O$-benzoyl- $\beta$-D-glucopyranosyl)formamide (6)}

Prepared from compound 1 ( $0.25 \mathrm{~g}, 0.40 \mathrm{mmol})$ and benzoyl chloride $(0.23 \mathrm{~mL}, 2.00 \mathrm{mmol})$ according to general procedure I (reaction time: $3 \mathrm{~d}$ ). Purified by column chromatography (1:3 EtOAc/hexane) to give $0.01 \mathrm{~g}(26 \%)$ white amorphous solid. $\mathrm{R}_{\mathrm{f}}=0.48(1: 1$ EtOAc/hexane); $[\alpha]_{\mathrm{D}}+88\left(\mathrm{c} 0.50, \mathrm{CHCl}_{3}\right) ;{ }^{1} \mathrm{H} \mathrm{NMR}\left(400 \mathrm{MHz}, \mathrm{CDCl}_{3}\right) \delta(\mathrm{ppm}): 8.12(1 \mathrm{H}, \mathrm{d}$, $J=7.4 \mathrm{~Hz}$, aromatics), $7.92-7.84(7 \mathrm{H}, \mathrm{m}$, aromatics $), 7.75(3 \mathrm{H}, \mathrm{d}, J=7.5 \mathrm{~Hz}$, aromatics $)$, 7.48-7.24 (19H, m, aromatics), 6.13 (1H, pseudo t, $J=9.6 \mathrm{~Hz}, \mathrm{H}-2$ ' or H-3' or H-4'), 5.94 (1H, pseudo t, $J=9.4 \mathrm{~Hz}, \mathrm{H}-2$ ' or H-3' or H-4'), 5.76 (1H, pseudo t, $J=9.5 \mathrm{~Hz}, \mathrm{H}-2$ ' or H-3' 
or H-4'), $5.11\left(1 \mathrm{H}, \mathrm{d}, J=9.9 \mathrm{~Hz}, \mathrm{H}-1^{\prime}\right), 4.35$ (1H, dd, $J=12.5,2.3 \mathrm{~Hz}, \mathrm{H}-6$ 'b), 4.26-4.19 (2H, m, H-6'a and H-5'); ${ }^{13} \mathrm{C}$ NMR (100 MHz, $\left.\mathrm{CDCl}_{3}\right) \delta(\mathrm{ppm}): 172.1,172.1,171.6,166.0$, 165.9, 165.2, $164.9(\mathrm{C}=\mathrm{O}), 133.9-128.4$ (aromatics), 77.1, 76.3, 74.5, 69.4, 68.6 (C-1'-C-5'), $62.6\left(\mathrm{C}-6\right.$ '). ESI-MS positive mode $(\mathrm{m} / \mathrm{z})$ : calcd for $\mathrm{C}_{49} \mathrm{H}_{37} \mathrm{NNaO}_{12}\left([\mathrm{M}+\mathrm{Na}]^{+}\right)$: 854.221. Found: 854.22. Anal. Calcd for $\mathrm{C}_{49} \mathrm{H}_{37} \mathrm{NO}_{12}$ : C, 70.75; H, 4.48; N, 1.68. Found: C, 70.99; H, $4.53 ; \mathrm{N}, 1.61$.

\section{$N, N$-Bis-(1-naphthoyl)- $C$-(2,3,4,6-tetra- $O$-benzoyl- $\beta$-D-glucopyranosyl)formamide (7)}

Prepared from compound $1(0.20 \mathrm{~g}, 0.32 \mathrm{mmol})$ and naphthoyl chloride $(0.24 \mathrm{~mL}, 1.6 \mathrm{mmol})$ according to general procedure I (reaction time: $1 \mathrm{~d}$ ). Purified by column chromatography (1:1:3 Toluene/EtOAc/hexane) to give $0.05 \mathrm{~g}(15 \%)$ white amorphous solid. $\mathrm{R}_{\mathrm{f}}=0.28(3: 1: 1$ Toluene/EtOAc/hexane); $[\alpha]_{\mathrm{D}}+44\left(\mathrm{c} 0.61, \mathrm{CHCl}_{3}\right) ;{ }^{1} \mathrm{H} \mathrm{NMR}\left(400 \mathrm{MHz}, \mathrm{CDCl}_{3}\right) \delta$ (ppm): $8.14(2 \mathrm{H}, \mathrm{d}, J=8.2 \mathrm{~Hz}$, aromatics), 7.98-7.85 (8H, m, aromatics), $7.65(2 \mathrm{H}, \mathrm{d}, J=7.7 \mathrm{~Hz}$, aromatics), 7.56-7.22 (18H, m, aromatics), $7.13(2 \mathrm{H}, \mathrm{t}, J=7.7 \mathrm{~Hz}$, aromatics), 6.97 (2H, t, $J$ = 7.7 Hz, aromatics), 6.28 (1H, pseudo t, $J=9.6 \mathrm{~Hz}, \mathrm{H}-2$ ' or H-3' or H-4'), 5.99 (1H, pseudo t, $J=9.5 \mathrm{~Hz}, \mathrm{H}-2^{\prime}$ or $\mathrm{H}^{-3} 3^{\prime}$ or H-4'), $5.76\left(1 \mathrm{H}\right.$, pseudo t, $J=9.7 \mathrm{~Hz}, \mathrm{H}-2^{\prime}$ or $\mathrm{H}-3$ ' or $\left.\mathrm{H}-4^{\prime}\right)$, $5.19\left(1 \mathrm{H}, \mathrm{d}, J=9.8 \mathrm{~Hz}, \mathrm{H}-1^{\prime}\right), 4.50(1 \mathrm{H}, \mathrm{dd}, J=12.5,2.0 \mathrm{~Hz}, \mathrm{H}-6$ 'a $), 4.35(1 \mathrm{H}, \mathrm{dd}, J=12.5$, $5.2 \mathrm{~Hz}, \mathrm{H}-6$ 'b), $4.25\left(1 \mathrm{H}, \mathrm{ddd}, J=10.0,5.3,2.0 \mathrm{~Hz}, \mathrm{H}-5\right.$ ') ${ }^{13}{ }^{13} \mathrm{NMR}\left(100 \mathrm{MHz}, \mathrm{CDCl}_{3}\right) \delta$ (ppm): 171.7, 171.7, 171.7, 166.0, 166.0, 165.2, 165.0, (C=O), 133.7-124.2 (aromatics), 77.1, 77.0, 74.5, 69.7, 68.5 (C-1'-C-5'), 62.8 (C-6'). ESI-MS positive mode (m/z): calcd for $\mathrm{C}_{57} \mathrm{H}_{41} \mathrm{NNaO}_{12}\left([\mathrm{M}+\mathrm{Na}]^{+}\right)$: 954.253. Found: 954.25. Anal. Calcd for $\mathrm{C}_{57} \mathrm{H}_{41} \mathrm{NO}_{12}: \mathrm{C}, 73.46$; H, 4.43; N, 1.50. Found: C, 73.60; H, 4.59; N, 1.45 .

\section{Acetone $N^{2}$-(2,3,4,6-tetra- $O$-benzoyl- $\beta$-D-glucopyranosylcarbonyl)hydrazone (10)}

To a solution of imide $2(0.10 \mathrm{~g}, 0.15 \mathrm{mmol})$ in pyridine was added hydrazine hydrate $(9 \mu \mathrm{L}$, 
1.2 equiv., $0.18 \mathrm{mmol}$ ) and the reaction mixture was stirred at room temperature. After disappearance of the starting material (3h, TLC, 1:1 Acetone/hexane) the solvent was removed and the purification by column chromatography (1:3 Acetone/hexane) yielded $0.04 \mathrm{~g}$ $(39 \%)$ yellow syrup. $\mathrm{R}_{\mathrm{f}}=0.26(1: 1$ Acetone/hexane $) ;[\alpha]_{\mathrm{D}}-24\left(\mathrm{c} 0.29, \mathrm{CHCl}_{3}\right) ;{ }^{1} \mathrm{H} \mathrm{NMR}$ (400 $\left.\mathrm{MHz}, \mathrm{CDCl}_{3}\right) \delta(\mathrm{ppm}): 8.07(2 \mathrm{H}, \mathrm{dd}, J=8.2,1.1 \mathrm{~Hz}$, aromatics), 7.97-7.94 (4H, m, aromatics), $7.83(2 \mathrm{H}, \mathrm{dd}, J=8.2,1.1 \mathrm{~Hz}$, aromatics), 7.62-7.26 (12H, m, aromatics), 5.96 (1H, pseudo t, $J=9.2 \mathrm{~Hz}, \mathrm{H}-2^{\prime}$ or $\mathrm{H}-3$ ' or H-4'), 5.74 (1H, pseudo t, $J=9.5 \mathrm{~Hz}, \mathrm{H}-2$ ' or H-3' or H-4'), 5.71 (1H, pseudo t, $J=9.5 \mathrm{~Hz}, \mathrm{H}-2^{\prime}$ ' or H-3' or H-4'), $4.83(1 \mathrm{H}, \mathrm{dd}, J=12.5,2.4 \mathrm{~Hz}$, H-6'a ), 4.53 (1H, dd, $J=12.5,4.9 \mathrm{~Hz}, \mathrm{H}-6$ 'b), 4.44 (1H, d, $J=9.5 \mathrm{~Hz}, \mathrm{H}-1$ '), 4.21 (1H, ddd, $J=9.9,4.9,2.4 \mathrm{~Hz}, \mathrm{H}-5$ ') $2.05\left(3 \mathrm{H}, \mathrm{s}, \mathrm{CH}_{3}\right), 1.85\left(3 \mathrm{H}, \mathrm{s}, \mathrm{CH}_{3}\right) ;{ }^{13} \mathrm{C} \mathrm{NMR}\left(100 \mathrm{MHz}, \mathrm{CDCl}_{3}\right)$ $\delta(\mathrm{ppm}): 166.3,165.8,165.5,165.3,162.4(\mathrm{C}=\mathrm{O}), 157.3(\mathrm{C}=\mathrm{N}), 133.8-128.3$ (aromatics), 76.7, 76.3, 73.5, 70.2, 68.8 (C-1'-C-5'), 62.1 (C-6'), 25.6, $16.6\left(2 \times \mathrm{CH}_{3}\right)$. ESI-MS positive mode $(\mathrm{m} / \mathrm{z})$ : calcd for $\mathrm{C}_{38} \mathrm{H}_{34} \mathrm{~N}_{2} \mathrm{NaO}_{10}\left([\mathrm{M}+\mathrm{Na}]^{+}\right)$: 701.211. Found: 701.21. Anal. Calcd for $\mathrm{C}_{38} \mathrm{H}_{34} \mathrm{~N}_{2} \mathrm{O}_{10}$ : C, 67.25; H, 5.05; N, 4.13. Found: C, 67.54; H, 5.18; N, 4.05.

\section{$N$-Acetyl- $C$-(2,3,4,6-tetra- $O$-benzoyl- $\beta$-D-glucopyranosyl)thioformamide (12)}

Prepared from compound $11(1.00 \mathrm{~g}, 1.56 \mathrm{mmol})$ and acetyl chloride (168 $\mu \mathrm{L}, 2.35 \mathrm{mmol})$ according to general procedure II (reaction time: $1 \mathrm{~h}$ ). Purified by column chromatography $(1: 3 \mathrm{EtOAc} / \mathrm{hexane})$ to give $0.96 \mathrm{~g}(91 \%)$ red foam. $\mathrm{R}_{\mathrm{f}}=0.50(1: 1 \mathrm{EtOAc} / \mathrm{hexane}) ;[\alpha]_{\mathrm{D}}=$

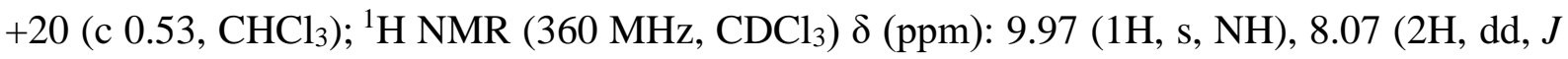
$=8.2,1.1 \mathrm{~Hz}$, aromatics $), 7.94-7.90(4 \mathrm{H}, \mathrm{m}$, aromatics $), 7.81(2 \mathrm{H}, \mathrm{dd}, J=8.2,1.1 \mathrm{~Hz}$, aromatics), 7.59-7.24 (12H, m, aromatics), $5.99(1 \mathrm{H}$, pseudo t, $J=9.3 \mathrm{~Hz}, \mathrm{H}-2$ ' or $\mathrm{H}-3$ ' or $\mathrm{H}-$ 4'), $5.76(1 \mathrm{H}$, pseudo t, $J=9.6 \mathrm{~Hz}, \mathrm{H}-2$ ' or H-3' or H-4'), $5.68(1 \mathrm{H}$, pseudo t, $J=9.3 \mathrm{~Hz}, \mathrm{H}-$ 2' or H-3' or H-4'), 4.88 (1H, d, $\left.J=9.0 \mathrm{~Hz}, \mathrm{H}-1^{\prime}\right), 4.72(1 \mathrm{H}, \mathrm{dd}, J=12.4,2.6 \mathrm{~Hz}, \mathrm{H}-6$ 'a), $4.57(1 \mathrm{H}, \mathrm{dd}, J=12.5,5.3 \mathrm{~Hz}, \mathrm{H}-6$ 'b), 4.30 (1H, ddd, $J=9.8,5.2,2.7 \mathrm{~Hz}, \mathrm{H}-5$ ') 2.42 (3H, s, 
$\left.\mathrm{CH}_{3}\right) ;{ }^{13} \mathrm{C} \mathrm{NMR}\left(90 \mathrm{MHz}, \mathrm{CDCl}_{3}\right) \delta(\mathrm{ppm}): 197.3(\mathrm{C}=\mathrm{S}), 170.5,166.4,165.7,165.3,165.2$ $(\mathrm{C}=\mathrm{O}), 133.6-128.2$ (aromatics), 84.9, 76.2, 73.4, 71.2, 69.0 (C-1'-C-5'), 62.9 (C-6'), 26.3 $\left(\mathrm{CH}_{3}\right)$. ESI-MS negative mode $(\mathrm{m} / \mathrm{z})$ : calcd for $\mathrm{C}_{37} \mathrm{H}_{30} \mathrm{NO}_{10} \mathrm{~S}\left([\mathrm{M}-\mathrm{H}]^{-}\right)$: 680.159. Found: 680.08. Anal. Calcd for $\mathrm{C}_{37} \mathrm{H}_{31} \mathrm{NO}_{10} \mathrm{~S}$ : C, 65.19; H, 4.58; N, 2.05; S, 4.70. Found: C, 65.33; H, 4.77; N, 2.09; S, 4.81 .

\section{$N$-Acetoxyacetyl- $C$ - $(2,3,4,6$-tetra- $O$-benzoyl- $\beta$-D-glucopyranosyl)thioformamide (13)}

Prepared from compound 11 (1.20 g, $1.87 \mathrm{mmol})$ and acetoxyacetyl chloride (303 $\mu \mathrm{L}, 2.81$ mmol) according to general procedure II (reaction time: $6 \mathrm{~h}$ ). Purified by column chromatography (1:3 EtOAc/hexane) to give $1.18 \mathrm{~g}(85 \%)$ red foam. $\mathrm{R}_{\mathrm{f}}=0.25(1: 2$ EtOAc/hexane); $[\alpha]_{\mathrm{D}}=+60\left(\mathrm{c} 0.45, \mathrm{CHCl}_{3}\right) ;{ }^{1} \mathrm{H} \mathrm{NMR}\left(400 \mathrm{MHz}, \mathrm{CDCl}_{3}\right) \delta(\mathrm{ppm}): 10.22(1 \mathrm{H}$, s, NH), $8.06(2 \mathrm{H}, \mathrm{d}, J=7.2 \mathrm{~Hz}$, aromatics), 7.93-7.90 (4H, m, aromatics), 7.81 (2H, d, $J=7.2$ Hz, aromatics), 7.60-7.25 (12H, m, aromatics), 5.99 (1H, pseudo t, $J=9.3 \mathrm{~Hz}, \mathrm{H}-2$ ' or H-3' or H-4'), 5.74 (1H, pseudo t, $J=9.6 \mathrm{~Hz}, \mathrm{H}-2^{\prime}$ or $\mathrm{H}^{-3}$ ' or $\mathrm{H}-4^{\prime}$ '), 5.69 (1H, pseudo t, $J=9.3$ Hz, H-2' or H-3' or H-4'), 5.04 (1H, d, $J=16.8 \mathrm{~Hz}, \mathrm{CH}_{2}$ ), 4.89 (1H, d, $J=9.3 \mathrm{~Hz}, \mathrm{H}-1^{\prime}$ '), $4.84\left(1 \mathrm{H}, \mathrm{d}, J=16.8 \mathrm{~Hz}, \mathrm{CH}_{2}\right), 4.67(1 \mathrm{H}, \mathrm{dd}, J=12.6,2.6 \mathrm{~Hz}, \mathrm{H}-6$ 'a), 4.58 (1H, dd, $J=12.5$, $5.6 \mathrm{~Hz}, \mathrm{H}-6$ 'b), 4.30 (1H, ddd, $J=9.6,5.5,2.5 \mathrm{~Hz}, \mathrm{H}-5$ '), $2.15\left(3 \mathrm{H}, \mathrm{s}, \mathrm{CH}_{3}\right) ;{ }^{13} \mathrm{C} \mathrm{NMR}(100$ $\left.\mathrm{MHz}, \mathrm{CDCl}_{3}\right) \delta(\mathrm{ppm}): 197.0(\mathrm{C}=\mathrm{S}), 169.9,167.3,166.4,165.7,165.3,165.2(\mathrm{C}=\mathrm{O}), 133.8-$ 128.5 (aromatics), 84.6, 76.4, 73.4, 71.2, 69.0 (C-1'-C-5'), $64.4\left(\mathrm{CH}_{2}\right), 63.0$ (C-6') 20.6 $\left(\mathrm{CH}_{3}\right)$. ESI-MS negative mode $(\mathrm{m} / \mathrm{z})$ : calcd for $\mathrm{C}_{39} \mathrm{H}_{32} \mathrm{NO}_{12} \mathrm{~S}\left([\mathrm{M}-\mathrm{H}]^{-}\right)$: 738.165. Found: 738.18. Anal. Calcd for $\mathrm{C}_{39} \mathrm{H}_{33} \mathrm{NO}_{12} \mathrm{~S}$ : C, 63.32; H, 4.50; N, 1.89; S, 4.33. Found: C, 63.67; H, 4.74; N, 1.87; S, 4.41.

\section{$N$-Pivaloyl- $C$-(2,3,4,6-tetra- $O$-benzoyl- $\beta$-D-glucopyranosyl)thioformamide (14)}

Prepared from compound 11 (1.10 g, $1.72 \mathrm{mmol})$ and pivaloyl chloride (317 $\mu \mathrm{L}, 2.58 \mathrm{mmol})$ 
according to general procedure II (reaction time: $1 \mathrm{~h}$ ). Purified by column chromatography (1:4 Acetone/hexane) to give $1.11 \mathrm{~g}(90 \%)$ red foam. $R_{\mathrm{f}}=0.50\left(1: 2\right.$ Acetone/hexane); $[\alpha]_{\mathrm{D}}=$ $+42\left(\mathrm{c} 0.53, \mathrm{CHCl}_{3}\right) ;{ }^{1} \mathrm{H} \mathrm{NMR}\left(400 \mathrm{MHz}, \mathrm{CDCl}_{3}\right) \delta(\mathrm{ppm}): 10.10(1 \mathrm{H}, \mathrm{s}, \mathrm{NH}), 8.06(2 \mathrm{H}, \mathrm{d}, J$ $=8.2 \mathrm{~Hz}$, aromatics), 7.95-7.88 (4H, m, aromatics), $7.82(2 \mathrm{H}, \mathrm{d}, J=8.2 \mathrm{~Hz}$, aromatics), 7.62$7.24\left(12 \mathrm{H}, \mathrm{m}\right.$, aromatics), $5.99\left(1 \mathrm{H}\right.$, pseudo t, $J=9.3 \mathrm{~Hz}, \mathrm{H}-2^{\prime}$ or $\mathrm{H}-3$ ' or H-4'), $5.80(1 \mathrm{H}$, pseudo t, $J=9.6 \mathrm{~Hz}, \mathrm{H}-2^{\prime}$ or $\mathrm{H}-3^{\prime}$ or $\left.\mathrm{H}-4^{\prime}\right), 5.71(1 \mathrm{H}$, pseudo t, $J=9.4 \mathrm{~Hz}, \mathrm{H}-2$ ' or H-3' or H4'), $5.04(1 \mathrm{H}, \mathrm{d}, J=9.0 \mathrm{~Hz}, \mathrm{H}-1$ '), 4.75 (1H, dd, $J=12.5,2.4 \mathrm{~Hz}, \mathrm{H}-6$ 'a), 4.54 (1H, dd, $J=$ 12.5, 4.7 Hz, H-6’b), 4.31 (1H, ddd, $J=9.8,4.5,2.5 \mathrm{~Hz}, \mathrm{H}-5$ '), $1.20\left(9 \mathrm{H}, \mathrm{s}, \mathrm{C}\left(\mathrm{CH}_{3}\right)_{3} ;{ }^{13} \mathrm{C}\right.$ NMR (100 MHz, $\left.\mathrm{CDCl}_{3}\right) \delta(\mathrm{ppm}): 196.8(\mathrm{C}=\mathrm{S}), 175.1,166.1,165.7,165.2,165.1(\mathrm{C}=\mathrm{O})$, 133.8-128.4 (aromatics), 84.2, 76.2, 73.7, 71.3, 68.9 (C-1'-C-5'), 62.4 (C-6'), 40.9 $\left(C\left(\mathrm{CH}_{3}\right)_{3}\right), 26.9\left(\mathrm{C}\left(\mathrm{CH}_{3}\right)_{3}\right)$. ESI-MS positive mode $(\mathrm{m} / \mathrm{z})$ : calcd for $\mathrm{C}_{40} \mathrm{H}_{38} \mathrm{NO}_{10} \mathrm{~S}\left([\mathrm{M}+\mathrm{H}]^{+}\right)$: 724.222. Found: 724.33. Anal. Calcd for $\mathrm{C}_{40} \mathrm{H}_{37} \mathrm{NO}_{10} \mathrm{~S}$ : C, 66.38; H, 5.15; N, 1.94; S, 4.43. Found: C, 66.52; H, 5.20; N, 1.90; S, 4.53.

\section{$N$-Phenylacetyl- $C$-(2,3,4,6-tetra- $O$-benzoyl- $\beta$-D-glucopyranosyl)thioformamide (15)}

Prepared from compound $11(1.10 \mathrm{~g}, 1.72 \mathrm{mmol})$ and 2-phenylacetyl chloride (341 $\mu \mathrm{L}, 2.58$ mmol) according to general procedure II (reaction time: 1d). Purified by column chromatography (1:5 EtOAc/hexane) to give $0.90 \mathrm{~g}(69 \%)$ red foam. $\mathrm{R}_{\mathrm{f}}=0.52(1: 1$ EtOAc/hexane); $[\alpha]_{\mathrm{D}}=+15\left(\mathrm{c} 0.51, \mathrm{CHCl}_{3}\right) ;{ }^{1} \mathrm{H} \mathrm{NMR}\left(360 \mathrm{MHz}, \mathrm{CDCl}_{3}\right) \delta(\mathrm{ppm}): 9.88(1 \mathrm{H}$, s, NH), $8.04(2 \mathrm{H}, \mathrm{d}, J=7.9 \mathrm{~Hz}$, aromatics), 7.92-7.89 (4H, m, aromatics), 7.80 (2H, d, $J=7.9$ Hz, aromatics), 7.59-7.26 (17H, m, aromatics), 5.92 (1H, pseudo t, J = 9.3 Hz, H-2' or H-3' or H-4'), 5.57 (2H, pseudo t, $J=9.6 \mathrm{~Hz}, \mathrm{H}-2^{\prime}$ ' and/or H-3' and/or H-4'), 4.85 (1H, d, $J=8.6$ Hz, H-1'), 4.55 (1H, dd, $J=12.3,2.5$ Hz, H-6'a), 4.37 (1H, dd, $J=12.4,5.8$ Hz, H-6'b), 4.22 $\left(1 \mathrm{H}, \mathrm{ddd}, J=9.1,5.6,2.5 \mathrm{~Hz}, \mathrm{H}-5\right.$ '), $3.90\left(2 \mathrm{H}, \mathrm{s}, \mathrm{PhCH}_{2}\right) ;{ }^{13} \mathrm{C} \mathrm{NMR}\left(90 \mathrm{MHz}, \mathrm{CDCl}_{3}\right) \delta$ (ppm): 196.6 (C=S), 169.3, 166.4, 165.7, 165.3, $165.2(\mathrm{C}=\mathrm{O}), 133.8-128.2$ (aromatics), 84.4, 
76.2, 73.5, 71.2, $69.2\left(\mathrm{C}-1^{\prime}-\mathrm{C}-5\right.$ '), $63.3\left(\mathrm{C}-6\right.$ ') $45.3\left(\mathrm{PhCH}_{2}\right)$. ESI-MS positive mode (m/z): calcd for $\mathrm{C}_{43} \mathrm{H}_{36} \mathrm{NO}_{10} \mathrm{~S}\left([\mathrm{M}+\mathrm{H}]^{+}\right)$: 758.206. Found: 758.25. Anal. Calcd for $\mathrm{C}_{43} \mathrm{H}_{35} \mathrm{NO}_{10} \mathrm{~S}: \mathrm{C}$, 68.15; H, 4.66; N, 1.85; S, 4.23. Found: C, 68.33; H, 4.80; N, 1.90; S, 4.39.

\section{$N$-(2,3,4,6-Tetra- $O$-benzoyl- $\beta$-D-glucopyranosylcarbonyl) thioacetamide (18)}

Prepared from compound $17(0.21 \mathrm{~g}, 0.32 \mathrm{mmol})$ and thioacetamide $(0.02 \mathrm{~g}, 0.64 \mathrm{mmol})$ according to general procedure III. Purified by column chromatography (1:3 EtOAc/hexane) to give $0.12 \mathrm{~g}(58 \%)$ yellow amorphous solid. $\mathrm{R}_{\mathrm{f}}=0.46(1: 2 \mathrm{EtOAc} / \mathrm{hexane}) ;[\alpha]_{\mathrm{D}}-146(\mathrm{c}$ 0.38, $\left.\mathrm{CHCl}_{3}\right) ;{ }^{1} \mathrm{H}$ NMR $\left(360 \mathrm{MHz}, \mathrm{CDCl}_{3}\right) \delta(\mathrm{ppm}): 10.17(1 \mathrm{H}, \mathrm{s}, \mathrm{NH}), 8.09-7.25(20 \mathrm{H}, \mathrm{m}$, aromatics), 6.00 (1H, pseudo t, $J=9.3 \mathrm{~Hz}, \mathrm{H}-2^{\prime}$ ' or $\mathrm{H}-3$ ' or $\mathrm{H}-4$ '), 5.72 (1H, pseudo t, $J=9.3$ Hz, H-2' or H-3' or H-4'), 5.70 (1H, pseudo t, $J=9.3$ Hz, H-2' or H-3' or H-4'), 4.76 (1H, dd, $J=12.5,2.7 \mathrm{~Hz}, \mathrm{H}-6$ 'a), $4.57(1 \mathrm{H}, \mathrm{dd}, J=12.5,5.1 \mathrm{~Hz}, \mathrm{H}-6$ 'b), $4.37(1 \mathrm{H}, \mathrm{d}, J=9.3 \mathrm{~Hz}$, H-1'), 4.28 (1H, ddd, $J=9.8,5.1,2.7 \mathrm{~Hz}, \mathrm{H}-5$ ') $2.88\left(3 \mathrm{H}, \mathrm{s}, \mathrm{CH}_{3}\right) ;{ }^{13} \mathrm{C}$ NMR $(90 \mathrm{MHz}$, $\left.\mathrm{CDCl}_{3}\right) \delta(\mathrm{ppm}): 210.6(\mathrm{C}=\mathrm{S}), 166.3,165.7,165.4,165.2,163.4(\mathrm{C}=\mathrm{O}), 133.8-128.5$ (aromatics), 76.6, 76.6, 73.0, 69.8, 68.8 (C-1'-C-5'), 62.5 (C-6'), $35.0\left(\mathrm{CH}_{3}\right)$;. ESI-MS positive mode $(\mathrm{m} / \mathrm{z})$ : calcd for $\mathrm{C}_{37} \mathrm{H}_{31} \mathrm{NNaO}_{10} \mathrm{~S}\left([\mathrm{M}+\mathrm{Na}]^{+}\right)$: 704.157. Found: 704.15. Anal. Calcd for $\mathrm{C}_{37} \mathrm{H}_{31} \mathrm{NO}_{10} \mathrm{~S}: \mathrm{C}, 65.19 ; \mathrm{H}, 4.58 ; \mathrm{N}, 2.05 ; \mathrm{S}, 4.70$. Found: $\mathrm{C}, 65.41 ; \mathrm{H}, 4.60 ; \mathrm{N}$, $2.03 ; \mathrm{S}, 4.78$.

\section{$N$-(2,3,4,6-Tetra- $O$-benzoyl- $\beta$-D-glucopyranosylcarbonyl) thiobenzamide (19)}

Prepared from compound $17(0.21 \mathrm{~g}, 0.32 \mathrm{mmol})$ and thiobenzamide $(0.04 \mathrm{~g}, 0.64 \mathrm{mmol})$ according to general procedure III. Purified by column chromatography (1:3 EtOAc/hexane) to give $0.17 \mathrm{~g}(71 \%)$ red solid. Mp: $124-127^{\circ} \mathrm{C} ; \mathrm{R}_{\mathrm{f}}=0.54(1: 2 \mathrm{EtOAc} / \mathrm{hexane}) ;[\alpha]_{\mathrm{D}}-204(\mathrm{c}$ 0.36, $\left.\mathrm{CHCl}_{3}\right) ;{ }^{1} \mathrm{H} \mathrm{NMR}\left(360 \mathrm{MHz}, \mathrm{CDCl}_{3}\right) \delta(\mathrm{ppm}): 10.38(1 \mathrm{H}, \mathrm{s}, \mathrm{NH}), 8.04-7.23(25 \mathrm{H}, \mathrm{m}$, aromatics), 6.00 (1H, pseudo t, $J=9.3 \mathrm{~Hz}, \mathrm{H}-2^{\prime}$ or $\mathrm{H}-3^{\prime}$ 'or $\left.\mathrm{H}-4^{\prime}\right), 5.84$ (1H, pseudo t, $J=9.3$ 
Hz, H-2' or H-3' or H-4'), 5.78 (1H, pseudo t, $J=9.3$ Hz, H-2' or H-3' or H-4'), $4.78(1 \mathrm{H}$, $\mathrm{dd}, J=12.3,1.8 \mathrm{~Hz}, \mathrm{H}-6$ 'a), $4.59(1 \mathrm{H}, \mathrm{dd}, J=12.3,5.0 \mathrm{~Hz}, \mathrm{H}-6$ 'b $), 4.51(1 \mathrm{H}, \mathrm{d}, J=9.3 \mathrm{~Hz}$, H-1'), $4.31\left(1 \mathrm{H}, \mathrm{ddd}, J=9.6,5.0,1.8 \mathrm{~Hz}, \mathrm{H}-5\right.$ '); ${ }^{13} \mathrm{C}$ NMR (90 MHz, $\left.\mathrm{CDCl}_{3}\right) \delta(\mathrm{ppm}): 202.2$ $(\mathrm{C}=\mathrm{S}), 166.3,165.7,165.3,165.2,163.5(\mathrm{C}=\mathrm{O}), 141.9-127.5$ (aromatics), 76.7, 76.6, 73.2, 69.6, 68.7 (C-1'-C-5'), 62.4 (C-6'). ESI-MS positive mode (m/z): calcd for $\mathrm{C}_{42} \mathrm{H}_{33} \mathrm{NNaO}_{10} \mathrm{~S}$ $\left([\mathrm{M}+\mathrm{Na}]^{+}\right):$766.172. Found: 766.17. Anal. Calcd for $\mathrm{C}_{42} \mathrm{H}_{33} \mathrm{NO}_{10} \mathrm{~S}: \mathrm{C}, 67.82 ; \mathrm{H}, 4.47 ; \mathrm{N}, 1.88$ S, 4.31. Found: C, 67.98; H, 4.56; N, 1.90; S, 4.30.

\section{3-(2',3',4',6'-Tetra- $O$-benzoyl- $\beta$-D-glucopyranosyl)-5-methyl-1,2,4-triazole (20)}

Prepared from compound $12(0.10 \mathrm{~g}, 0.15 \mathrm{mmol})$ and hydrazinium acetate $(16 \mathrm{mg}, 0.18$ mmol) according to general procedure IV. Purification by column chromatography (3:2 EtOAc/hexane) yielded $0.06 \mathrm{~g}(55 \%)$ pale yellow amorphous solid.

Prepared from compound $18(0.12 \mathrm{~g}, 0.18 \mathrm{mmol})$ and hydrazinium acetate $(19 \mathrm{mg}, 0.21$ mmol) according to general procedure IV. Purification by column chromatography $(3: 2$ EtOAc/hexane) yielded $0.07 \mathrm{~g}(57 \%)$ pale yellow amorphous solid.

The compound characterization data are identical with those reported ${ }^{20}$.

\section{3-(2',3',4',6'-Tetra-O-benzoyl- $\beta$-D-glucopyranosyl)-5-methyl-1-phenyl-1,2,4-triazole (21)}

Prepared from compound $12(0.35 \mathrm{~g}, 0.51 \mathrm{mmol})$ and phenylhydrazine $(61 \mu \mathrm{L}, 0.62 \mathrm{mmol})$ according to general procedure IV. Purified by column chromatography (1:2 Acetone/hexane) to give $0.27 \mathrm{~g}(71 \%)$ pale yellow amorphous solid. $\mathrm{R}_{\mathrm{f}}=0.42(1: 1$ Acetone/hexane $\left.) ; \alpha\right]_{\mathrm{D}}=$ $+12\left(\mathrm{c} 0.50, \mathrm{CHCl}_{3}\right) ;{ }^{1} \mathrm{H} \mathrm{NMR}\left(400 \mathrm{MHz}, \mathrm{CDCl}_{3}\right) \delta(\mathrm{ppm}): 8.00(2 \mathrm{H}, \mathrm{dd}, J=8.3,1.2 \mathrm{~Hz}$, aromatics), $7.92(2 \mathrm{H}, \mathrm{dd}, J=8.3,1.1 \mathrm{~Hz}$, aromatics), 7.88-7.85 (4H, m, aromatics), 7.51-7.21 (17H, m, aromatics), 6.17 (1H, pseudo t, $J=9.7 \mathrm{~Hz}, \mathrm{H}-2^{\prime}$ ' or $\mathrm{H}-3$ ' or H-4'), 6.07 (1H, pseudo t, $J=9.5 \mathrm{~Hz}, \mathrm{H}-2^{\prime}$ or $\mathrm{H}-3$ ' or H-4'), 5.88 (1H, pseudo t, $J=9.7 \mathrm{~Hz}, \mathrm{H}-2$ ' or H-3' or H-4'), 
$5.08\left(1 \mathrm{H}, \mathrm{d}, J=9.8 \mathrm{~Hz}, \mathrm{H}-1^{\prime}\right), 4.66(1 \mathrm{H}, \mathrm{dd}, J=12.3,3.1 \mathrm{~Hz}, \mathrm{H}-6$ 'a), 4.59 (1H, dd, $J=12.3$, $5.0 \mathrm{~Hz}, \mathrm{H}-6$ 'b), 4.38 (1H, ddd, $J=9.7,4.9,3.2 \mathrm{~Hz}, \mathrm{H}-5$ '), $2.46\left(3 \mathrm{H}, \mathrm{s}, \mathrm{CH}_{3}\right) ;{ }^{13} \mathrm{C} \mathrm{NMR}(100$ $\left.\mathrm{MHz}, \mathrm{CDCl}_{3}\right) \delta(\mathrm{ppm}): 166.3,165.9,165.2,164.7(\mathrm{C}=\mathrm{O}), 158.5,153.4$ (triazole $\left.\mathrm{C}-3, \mathrm{C}-5\right)$, 137.1 (q, Ph), 133.4-124.5 (aromatics), 76.8, 74.7, 74.6, 71.5, 69.8 (C-1'-C-5'), 63.7 (C-6’), $13.3\left(\mathrm{CH}_{3}\right)$. ESI-MS positive mode $(\mathrm{m} / \mathrm{z})$ : calcd for $\mathrm{C}_{43} \mathrm{H}_{36} \mathrm{~N}_{3} \mathrm{O}_{9}\left([\mathrm{M}+\mathrm{H}]^{+}\right)$: 738.245. Found: 738.25. Anal. Calcd for $\mathrm{C}_{43} \mathrm{H}_{35} \mathrm{~N}_{3} \mathrm{O}_{9}: \mathrm{C}, 70.01 ; \mathrm{H}, 4.78 ; \mathrm{N}, 5.70$. Found: $\mathrm{C}, 70.27 ; \mathrm{H}, 4.90 ; \mathrm{N}$, 5.65 .

\section{3-(2',3',4',6'-Tetra-O-benzoyl- $\beta$-D-glucopyranosyl)-1-(2-hydroxyethyl)-5-methyl-1,2,4-}

\section{triazole (22)}

Prepared from compound $12(0.33 \mathrm{~g}, 0.49 \mathrm{mmol})$ and 2-hydroxyethylhydrazine (40 $\mu \mathrm{L}, 0.58$ mmol) according to general procedure IV. Purified by column chromatography (1:2 Acetone/hexane) to give $0.24 \mathrm{~g}(70 \%)$ pale yellow amorphous solid. $\mathrm{R}_{\mathrm{f}}=0.15(4: 1$ EtOAc/hexane); $[\alpha]_{\mathrm{D}}=+21\left(\mathrm{c} 0.55, \mathrm{CHCl}_{3}\right) ;{ }^{1} \mathrm{H} \mathrm{NMR}\left(400 \mathrm{MHz}, \mathrm{CDCl}_{3}\right) \delta(\mathrm{ppm}): 8.00(2 \mathrm{H}$, $\mathrm{dd}, J=8.2,1.0 \mathrm{~Hz}$, aromatics), $7.92(2 \mathrm{H}, \mathrm{dd}, J=8.2,1.1 \mathrm{~Hz}$, aromatics), 7.82-7.85 (4H, m, aromatics), 7.51-7.23 (12H, m, aromatics), 6.09-5.99 (2H, m, H-2' and/or H-3' and/or H-4'), $5.85\left(1 \mathrm{H}\right.$, pseudo t, $J=9.5 \mathrm{~Hz}, \mathrm{H}-2^{\prime}$ or $\mathrm{H}^{\prime} 3^{\prime}$ or $\left.\mathrm{H}^{-4}{ }^{\prime}\right), 4.96\left(1 \mathrm{H}, \mathrm{d}, J=9.3 \mathrm{~Hz}, \mathrm{H}-1^{\prime}\right), 4.65$ $(1 \mathrm{H}, \mathrm{dd}, J=12.33 .0 \mathrm{~Hz}, \mathrm{H}-6$ 'a), 4.57 (1H, dd, $J=12.3,5.1 \mathrm{~Hz}, \mathrm{H}-6$ 'b), 4.35 (1H, ddd, $J=$ 9.6, 5.0, 3.2 Hz, H-5'), $4.00\left(2 \mathrm{H}, \mathrm{t}, J=4.7 \mathrm{~Hz}, \mathrm{CH}_{2}\right), 3.95-3.90\left(1 \mathrm{H}, \mathrm{m}, \mathrm{CH}_{2}\right), 3.75-3.71(1 \mathrm{H}$, m, $\left.\mathrm{CH}_{2}\right), 3.13\left(1 \mathrm{H}\right.$, br s, OH), $2.34\left(3 \mathrm{H}, \mathrm{s}, \mathrm{CH}_{3}\right) ;{ }^{13} \mathrm{C} \mathrm{NMR}\left(100 \mathrm{MHz}, \mathrm{CDCl}_{3}\right) \delta(\mathrm{ppm})$ : 166.2, 165.9, 165.3, $165.2(\mathrm{C}=\mathrm{O}), 158.0,154.1$ (triazole C-3, C-5), 133.4-128.3 (aromatics), 76.7, 74.7, 74.3, 72.1, $69.7\left(\mathrm{C}-1^{\prime}{ }^{-} \mathrm{C}-5^{\prime}\right), 63.6\left(\mathrm{C}-6\right.$ ') $, 60.6,50.5\left(2 \times \mathrm{CH}_{2}\right), 11.9\left(\mathrm{CH}_{3}\right)$. ESIMS positive mode $(\mathrm{m} / \mathrm{z})$ : calcd for $\mathrm{C}_{39} \mathrm{H}_{36} \mathrm{~N}_{3} \mathrm{O}_{10}\left([\mathrm{M}+\mathrm{H}]^{+}\right)$: 706.240. Found: 706.28. Anal. Calcd for $\mathrm{C}_{39} \mathrm{H}_{35} \mathrm{~N}_{3} \mathrm{O}_{10}:$ C, 66.38; H, 5.00; N, 5.95. Found: C, 66.40; H, 5.09; N, 6.03. 
3-(2',3',4',6'-Tetra-O-benzoyl- $\beta$-D-glucopyranosyl)-5-methyl-1-tosyl-1,2,4-triazole (23)

Prepared from compound $12(0.10 \mathrm{~g}, 0.15 \mathrm{mmol})$ and tosylhydrazine (34 mg, $0.18 \mathrm{mmol})$ according to general procedure IV. Purification by column chromatography (1:2 EtOAc/hexane) yielded $0.08 \mathrm{~g}(63 \%)$ pale yellow amorphous solid. The compound characterization data are identical with those reported. ${ }^{20}$

\section{5-(Acetoxymethyl)-3-(2',3',4',6'-tetra- $O$-benzoyl- $\beta$-D-glucopyranosyl)-1,2,4-triazole (24)}

Prepared from compound $13(0.10 \mathrm{~g}, 0.14 \mathrm{mmol})$ hydrazinium acetate $(15 \mathrm{mg}, 0.16 \mathrm{mmol})$ according to general procedure IV. Purification by column chromatography (1:2 EtOAc/hexane) yielded $0.09 \mathrm{~g}(92 \%)$ pale yellow amorphous solid. The compound characterization data are identical with those reported. ${ }^{20}$

\section{5-(Acetoxymethyl)-3-(2',3',4',6'-tetra- $O$-benzoyl- $\beta$-D-glucopyranosyl)-1-phenyl-1,2,4- triazole (25)}

Prepared from compound $13(0.30 \mathrm{~g}, 0.41 \mathrm{mmol})$ and phenylhydrazine (48 $\mu \mathrm{L}, 0.49 \mathrm{mmol})$ according to general procedure IV. Purified by column chromatography (1:2 EtOAc/hexane) to give $0.22 \mathrm{~g}(70 \%)$ pale yellow amorphous solid. $\mathrm{R}_{\mathrm{f}}=0.28(1: 1$ EtOAc/hexane $) ;[\alpha]_{\mathrm{D}}=+4$ (c $\left.0.48, \mathrm{CHCl}_{3}\right) ;{ }^{1} \mathrm{H} \mathrm{NMR}\left(400 \mathrm{MHz}, \mathrm{CDCl}_{3}\right) \delta(\mathrm{ppm}): 8.01(2 \mathrm{H}, \mathrm{dd}, J=8.2,1.1 \mathrm{~Hz}$, aromatics), $7.92(2 \mathrm{H}, \mathrm{dd}, J=8.2,1.0$, aromatics), 7.88-7.84 (4H, m, aromatics), 7.51-7.23 (17H, m, aromatics), 6.20 (1H, pseudo t, $J=9.7 \mathrm{~Hz}, \mathrm{H}-2^{\prime}$ ' or $\mathrm{H}-3$ ' or H-4'), 6.09 (1H, pseudo t, $J=9.5 \mathrm{~Hz}, \mathrm{H}-2^{\prime}$ or $\mathrm{H}_{-3}{ }^{\prime}$ or $\left.\mathrm{H}-4^{\prime}\right), 5.90\left(1 \mathrm{H}\right.$, pseudo t, $J=9.7 \mathrm{~Hz}, \mathrm{H}-2^{\prime}$ or $\mathrm{H}-3^{\prime}$ or $\left.\mathrm{H}-4^{\prime}\right)$, $5.15(1 \mathrm{H}, \mathrm{d}, J=9.8 \mathrm{~Hz}, \mathrm{H}-1$ ' $), 5.12\left(2 \mathrm{H}, \mathrm{s}, \mathrm{CH}_{2}\right), 4.68(1 \mathrm{H}, \mathrm{dd}, J=12.3,3.0 \mathrm{~Hz}, \mathrm{H}-6$ 'a), 4.59 (1H, dd, $J=12.3,5.0 \mathrm{~Hz}, \mathrm{H}-6$ 'b), 4.40 (1H, ddd, $J=9.7,4.8,3.1 \mathrm{~Hz}, \mathrm{H}-5$ ') 1.96 (3H, s, $\left.\mathrm{CH}_{3}\right) ;{ }^{13} \mathrm{C} \mathrm{NMR}\left(100 \mathrm{MHz}, \mathrm{CDCl}_{3}\right) \delta(\mathrm{ppm}): 169.8,166.2,165.9,165.2,164.6(\mathrm{C}=\mathrm{O}), 159.2$, 151.2 (triazole C-3, C-5), 136.5 (q, Ph), 133.4-124.6 (aromatics), 76.8, 74.5, 74.5, 71.5, 69.7 
(C-1'-C-5'), 63.5 (C-6'), $56.3\left(\mathrm{CH}_{2}\right), 20.3\left(\mathrm{CH}_{3}\right)$. ESI-MS positive mode (m/z): calcd for $\mathrm{C}_{45} \mathrm{H}_{38} \mathrm{~N}_{3} \mathrm{O}_{11}\left([\mathrm{M}+\mathrm{H}]^{+}\right):$796.251. Found: 796.25. Anal. Calcd for $\mathrm{C}_{45} \mathrm{H}_{37} \mathrm{~N}_{3} \mathrm{O}_{11}: \mathrm{C}, 67.92 ; \mathrm{H}$, 4.69; N, 5.28. Found: C, 68.11; H, 4.82; N, 5.22.

\section{3-(2',3',4',6'-Tetra-O-benzoyl- $\beta$-D-glucopyranosyl)-1-(2-hydroxyethyl)-5-}

\section{(hydroxymethyl)-1,2,4-triazole (26)}

Prepared from compound $13(0.30 \mathrm{~g}, 0.41 \mathrm{mmol})$ and 2-hydroxyethylhydrazine (33 $\mu \mathrm{L}, 0.49$ mmol) according to general procedure IV. Purified by column chromatography (1:2 EtOAc/hexane) to give $0.19 \mathrm{~g}(65 \%)$ pale yellow amorphous solid. $\mathrm{R}_{\mathrm{f}}=0.45(2: 1$ Acetone/hexane); $[\alpha]_{\mathrm{D}}=+6\left(\mathrm{c} 0.47, \mathrm{CHCl}_{3}\right) ;{ }^{1} \mathrm{H} \mathrm{NMR}\left(400 \mathrm{MHz}, \mathrm{CDCl}_{3}\right) \delta(\mathrm{ppm}): 7.98(2 \mathrm{H}$, $\mathrm{dd}, J=8.2,1.2 \mathrm{~Hz}$, aromatics), $7.91(2 \mathrm{H}, \mathrm{dd}, J=8.2,1.2$, aromatics), 7.84-7.79 (4H, m, aromatics), 7.51-7.23 (12H , m, aromatics), $6.05(1 \mathrm{H}$, pseudo t, $J=9.5 \mathrm{~Hz}, \mathrm{H}-2$ ' or $\mathrm{H}-3$ ' or H-4'), $5.96\left(1 \mathrm{H}\right.$, pseudo t, $J=9.6 \mathrm{~Hz}, \mathrm{H}-2^{\prime}$ or $\mathrm{H}^{-3}{ }^{\prime}$ or $\left.\mathrm{H}-4^{\prime}\right), 5.85(1 \mathrm{H}$, pseudo t, $J=9.6 \mathrm{~Hz}$, H-2' or H-3' or H-4'), 4.98 (1H, d, $J=9.6$ Hz, H-1'), 4.67-4.62 (1H, m, H-6'a), 4.62 (2H, s, $\left.\mathrm{CH}_{2}\right), 4.53(1 \mathrm{H}, \mathrm{dd}, J=12.3,5.0 \mathrm{~Hz}, \mathrm{H}-6$ 'b), 4.34 (1H, ddd, $J=9.7,4.8,3.1 \mathrm{~Hz}, \mathrm{H}-5$ '), 4.13 $\left(2 \mathrm{H}, \mathrm{t}, J=4.6 \mathrm{~Hz}, \mathrm{CH}_{2}\right), 3.82-3.77\left(1 \mathrm{H}, \mathrm{m}, \mathrm{CH}_{2}\right), 3.71-3.65\left(1 \mathrm{H}, \mathrm{m}, \mathrm{CH}_{2}\right) ;{ }^{13} \mathrm{C} \mathrm{NMR}(100$ $\left.\mathrm{MHz}, \mathrm{CDCl}_{3}\right) \delta(\mathrm{ppm}): 166.3,165.9,165.3,165.3(\mathrm{C}=\mathrm{O}), 158.0,156.5$ (triazole C-3, C-5), 133.5-128.3 (aromatics), 76.7, 74.4, 74.3, 71.9, 69.7 (C-1'-C-5'), 63.5 (C-6'), 60.6, 55.0, $51.1\left(3 \times \mathrm{CH}_{2}\right)$. ESI-MS negative mode $(\mathrm{m} / \mathrm{z})$ : calcd for $\mathrm{C}_{41} \mathrm{H}_{38} \mathrm{~N}_{3} \mathrm{O}_{13}\left([\mathrm{M}+\mathrm{OAc}]^{-}\right)$: 780.240 . Found: 780.27. Anal. Calcd for $\mathrm{C}_{39} \mathrm{H}_{35} \mathrm{~N}_{3} \mathrm{O}_{11}$ : C, 64.90; H, 4.89; N, 5.82. Found: C, 65.22; H, 4.99; N, 5.93.

\section{3-(2',3',4',6'-Tetra-O-benzoyl- $\beta$-D-glucopyranosyl)-5-(tert-butyl)-1,2,4-triazole (27)}

Prepared from compound $14(0.10 \mathrm{~g}, 0.14 \mathrm{mmol})$ hydrazinium acetate $(15 \mathrm{mg}, 0.17 \mathrm{mmol})$ according to general procedure IV. Purification by column chromatography (1:2 
EtOAc/hexane) yielded 0.08g (79\%) pale yellow amorphous solid. The compound characterization data are identical with those reported. ${ }^{20}$

\section{3-(2',3',4',6'-Tetra- $O$-benzoyl- $\beta$-D-glucopyranosyl)-5-(tert-butyl)-1-phenyl-1,2,4-triazole} (28)

Prepared from compound $14(0.30 \mathrm{~g}, 0.41 \mathrm{mmol})$ and phenylhydrazine (48 $\mu \mathrm{L}, 0.49 \mathrm{mmol})$ according to general procedure IV. Purified by column chromatography (1:4 EtOAc/hexane) to give $0.21 \mathrm{~g}(66 \%)$ pale yellow amorphous solid. $\mathrm{R}_{\mathrm{f}}=0.55(1: 1$ EtOAc/hexane $\left.) ; \alpha\right]_{\mathrm{D}}=+9$ (c $\left.0.49, \mathrm{CHCl}_{3}\right) ;{ }^{1} \mathrm{H} \mathrm{NMR}\left(400 \mathrm{MHz}, \mathrm{CDCl}_{3}\right) \delta(\mathrm{ppm}): 8.01(2 \mathrm{H}, \mathrm{dd}, J=8.2,1.2 \mathrm{~Hz}$, aromatics), 7.94-7.85 (6H, m, aromatics), 7.51-7.25 (15H , m, aromatics), 7.15-7.13 (2H, m, aromatics), 6.13 (1H, pseudo t, $J=9.7 \mathrm{~Hz}, \mathrm{H}-2^{\prime}$ ' or H-3' or H-4'), 6.05 (1H, pseudo t, $J=9.5$ Hz, H-2' or H-3' or H-4'), 5.89 (1H, pseudo t, $J=9.6$ Hz, H-2' or H-3' or H-4'), 5.05 (1H, d, $\left.J=9.7 \mathrm{~Hz}, \mathrm{H}-1^{\prime}\right), 4.66(1 \mathrm{H}, \mathrm{dd}, J=12.3,3.1 \mathrm{~Hz}, \mathrm{H}-6$ 'a $), 4.59(1 \mathrm{H}, \mathrm{dd}, J=12.3,5.0 \mathrm{~Hz}, \mathrm{H}-$ 6’b), $4.36\left(1 \mathrm{H}, \mathrm{ddd}, J=9.6,4.7,3.3 \mathrm{~Hz}, \mathrm{H}-5\right.$ ') $1.09\left(9 \mathrm{H}, \mathrm{s}, \mathrm{C}\left(\mathrm{CH}_{3}\right)_{3}\right) ;{ }^{13} \mathrm{C} \mathrm{NMR}(100 \mathrm{MHz}$, $\left.\mathrm{CDCl}_{3}\right) \delta(\mathrm{ppm}): 166.3,165.9,165.3,164.7(\mathrm{C}=\mathrm{O}), 164.1,157.1$ (triazole C-3, C-5), $139.5(\mathrm{q}$, $\mathrm{Ph}), 133.4-127.9$ (aromatics), 77.0, 74.9, 74.5, 72.1, 69.9 (C-1'-C-5'), 63.7 (C-6'), 33.5 (C(CH3)3), 29.7 $\left(\mathrm{C}\left(\mathrm{CH}_{3}\right)_{3}\right)$. ESI-MS positive mode $(\mathrm{m} / \mathrm{z})$ : calcd for $\mathrm{C}_{46} \mathrm{H}_{42} \mathrm{~N}_{3} \mathrm{O}_{9}\left([\mathrm{M}+\mathrm{H}]^{+}\right)$: 780.292. Found: 780.30. Anal. Calcd for $\mathrm{C}_{46} \mathrm{H}_{41} \mathrm{~N}_{3} \mathrm{O}_{9}$ : C, 70.85; H, 5.30; N, 5.39. Found: C, $70.91 ; \mathrm{H}, 5.42 ; \mathrm{N}, 5.34$

\section{3-(2',3', $4^{\prime}, 6^{\prime}$-Tetra- $O$-benzoyl- $\beta$-D-glucopyranosyl)-5-(tert-butyl)-1-(2-hydroxyethyl)-}

\section{1,2,4-triazole (29)}

Prepared from compound $14(0.30 \mathrm{~g}, 0.41 \mathrm{mmol})$ and 2-hydroxyethylhydrazine (33 $\mu \mathrm{L}, 0.49$ mmol) according to general procedure IV. Purified by column chromatography $(1: 1$ EtOAc/hexane) to give $0.21 \mathrm{~g}(66 \%)$ white amorphous solid. $\mathrm{R}_{\mathrm{f}}=0.20(1: 1 \mathrm{EtOAc} / \mathrm{hexane})$; 
$[\alpha]_{\mathrm{D}}=+29\left(\mathrm{c} 0.56, \mathrm{CHCl}_{3}\right) ;{ }^{1} \mathrm{H} \mathrm{NMR}\left(400 \mathrm{MHz}, \mathrm{CDCl}_{3}\right) \delta(\mathrm{ppm}): 8.01(2 \mathrm{H}, \mathrm{dd}, J=8.2,1.1$ $\mathrm{Hz}$, aromatics), $7.93(2 \mathrm{H}, \mathrm{dd}, J=8.2,1.0$, aromatics), 7.88-7.80 (4H, m, aromatics), 7.52$7.22(12 \mathrm{H}, \mathrm{m}$, aromatics $), 6.11\left(1 \mathrm{H}\right.$, pseudo t, $J=9.6 \mathrm{~Hz}, \mathrm{H}-2^{\prime}$ or $\mathrm{H}-3$ ' or H-4'), $6.06(1 \mathrm{H}$, pseudo t, $J=9.6 \mathrm{~Hz}, \mathrm{H}-2^{\prime}$ or $\mathrm{H}-3^{\prime}$ ' or H-4'), 5.87 (1H, pseudo t, $J=9.5 \mathrm{~Hz}, \mathrm{H}-2$ ' or H-3' or H4'), $5.00(1 \mathrm{H}, \mathrm{d}, J=9.4 \mathrm{~Hz}, \mathrm{H}-1$ '), 4.66 (1H, dd, $J=12.3,2.9 \mathrm{~Hz}, \mathrm{H}-6$ 'a), 4.57 (1H, dd, $J=$ 12.3, 5.1 Hz, H-6'b), 4.35 (1H, ddd, $J=9.6,5.0,3.2 \mathrm{~Hz}, \mathrm{H}-5$ ') $4.32-4.27\left(1 \mathrm{H}, \mathrm{m}, \mathrm{CH}_{2}\right)$, 4.22-4.14 (2H, m, CH 2$), 3.91-3.85\left(1 \mathrm{H}, \mathrm{m}, \mathrm{CH}_{2}\right), 1.25\left(9 \mathrm{H}, \mathrm{s}, \mathrm{C}\left(\mathrm{CH}_{3}\right)_{3}\right) ;{ }^{13} \mathrm{C} \mathrm{NMR}(100$ $\left.\mathrm{MHz}, \mathrm{CDCl}_{3}\right) \delta(\mathrm{ppm}): 166.2,165.8,165.4,165.2(\mathrm{C}=\mathrm{O}), 163.3,156.7$ (triazole $\left.\mathrm{C}-3, \mathrm{C}-5\right)$, 133.4-128.3 (aromatics), 76.8, 74.8, 74.2, 72.2, 69.8 (C-1'-C-5'), 63.6 (C-6'), 60.8, 52.6 (2 × $\left.\mathrm{CH}_{2}\right), 32.4\left(\mathrm{C}\left(\mathrm{CH}_{3}\right)_{3}\right), 29.1\left(\mathrm{C}\left(\mathrm{CH}_{3}\right)_{3}\right)$. ESI-MS positive mode $(\mathrm{m} / \mathrm{z})$ : calcd for $\mathrm{C}_{42} \mathrm{H}_{42} \mathrm{~N}_{3} \mathrm{O}_{10}$ $\left([\mathrm{M}+\mathrm{H}]^{+}\right):$748.287. Found: 748.35. Anal. Calcd for $\mathrm{C}_{42} \mathrm{H}_{41} \mathrm{~N}_{3} \mathrm{O}_{10}: \mathrm{C}, 67.46 ; \mathrm{H}, 5.53 ; \mathrm{N}, 5.62$. Found: C, 67.78; H, 5.80; N, 5.79.

\section{3-(2',3',4',6'-Tetra- $O$-benzoyl- $\beta$-D-glucopyranosyl)-5-benzyl-1,2,4-triazole (30)}

Prepared from compound $15(0.30 \mathrm{~g}, 0.39 \mathrm{mmol})$ and hydrazinium acetate $(44 \mathrm{mg}, 0.48$ mmol) according to general procedure IV. Purified by column chromatography $(1: 2$ EtOAc/hexane) to give $0.22 \mathrm{~g}(75 \%)$ white amorphous solid. $\mathrm{R}_{\mathrm{f}}=0.45$ (2:1 Acetone/hexane); $[\alpha]_{\mathrm{D}}=+15\left(\mathrm{c} 0.54, \mathrm{CHCl}_{3}\right) ;{ }^{1} \mathrm{H} \mathrm{NMR}\left(360 \mathrm{MHz}, \mathrm{CDCl}_{3}\right) \delta(\mathrm{ppm}): 7.94-7.90(4 \mathrm{H}, \mathrm{m}$, aromatics), 7.79-7.77 (4H, m, aromatics), 7.50-7.07 (17H, m, aromatics), 6.19 (1H, pseudo t, $J=9.7 \mathrm{~Hz}, \mathrm{H}-2^{\prime}$ 'or H-3' or H-4'), 6.05 (1H, pseudo t, $J=9.5 \mathrm{~Hz}, \mathrm{H}-2$ ' or H-3' or H-4'), 5.91 (1H, pseudo t, $J=9.7 \mathrm{~Hz}, \mathrm{H}-2$ ' or H-3' or H-4'), $5.12\left(1 \mathrm{H}, \mathrm{d}, J=9.9 \mathrm{~Hz}, \mathrm{H}-1^{\prime}\right), 4.56-4.54$ (2H, m, H-6'a, H-6’b), 4.59 (1H, dd, $J=12.3,5.0$ Hz, H-6'b), 4.38-4.33 (1H, m, H-5'), 4.02 $\left(2 \mathrm{H}, \mathrm{s}, \mathrm{PhCH}_{2}\right) ;{ }^{13} \mathrm{C} \mathrm{NMR}\left(90 \mathrm{MHz}, \mathrm{CDCl}_{3}\right) \delta(\mathrm{ppm}): 166.4,166.1,165.3,165.1(\mathrm{C}=\mathrm{O})$, 158.4, 157.8 (triazole C-3, C-5), 135.7 (q, Ph), 133.5-127.2 (aromatics), 76.9, 74.6, 74.4, 71.5, $69.8\left(\mathrm{C}-1^{\prime}-\mathrm{C}-5^{\prime}\right), 63.6\left(\mathrm{C}-6\right.$ ') $33.2\left(\mathrm{PhCH}_{2}\right)$. ESI-MS negative mode $(\mathrm{m} / \mathrm{z})$ : calcd for 
$\mathrm{C}_{43} \mathrm{H}_{34} \mathrm{~N}_{3} \mathrm{O}_{9}\left([\mathrm{M}-\mathrm{H}]^{-}\right):$736.229. Found: 736.23. Anal. Calcd for $\mathrm{C}_{43} \mathrm{H}_{35} \mathrm{~N}_{3} \mathrm{O}$ : $\mathrm{C}, 70.01 ; \mathrm{H}$, 4.78; N, 5.70. Found: C, 70.22; H, 4.87; N, 5.75.

\section{3-(2',3',4',6'-Tetra- $O$-benzoyl- $\beta$-D-glucopyranosyl)-5-benzyl-1-phenyl-1,2,4-triazole (31)}

Prepared from compound $15(0.28 \mathrm{~g}, 0.37 \mathrm{mmol})$ and phenylhydrazine $(44 \mu \mathrm{L}, 0.45 \mathrm{mmol})$ according to general procedure IV. Purified by column chromatography (1:3 EtOAc/hexane) to give $0.21 \mathrm{~g}(71 \%)$ pale orange amorphous solid. $R_{\mathrm{f}}=0.38(1: 1$ Acetone/hexane $) ;[\alpha]_{\mathrm{D}}=+4$ (c 0.54, $\left.\mathrm{CHCl}_{3}\right) ;{ }^{1} \mathrm{H} \mathrm{NMR}\left(400 \mathrm{MHz}, \mathrm{CDCl}_{3}\right) \delta$ (ppm): 8.03-8.00 (2H, m, aromatics), 7.947.92 (2H, m, aromatics), 7.89-7.83 (4H, m, aromatics), 7.51-7.24 (16H, m, aromatics), 7.10$7.04(5 \mathrm{H}, \mathrm{m}$, aromatics), $6.95(2 \mathrm{H}, \mathrm{dd}, J=7.4,1.9 \mathrm{~Hz}$, aromatics), 6.25 (1H, pseudo t, $J=9.8$ Hz, H-2' or H-3' or H-4'), 6.08 (1H, pseudo t, $J=9.5$ Hz, H-2' or H-3' or H-4'), $5.91(1 \mathrm{H}$, pseudo t, $J=9.7 \mathrm{~Hz}, \mathrm{H}-2$ ' or H-3' or H-4'), $5.12(1 \mathrm{H}, \mathrm{d}, J=9.9 \mathrm{~Hz}, \mathrm{H}-1$ '), $4.68(1 \mathrm{H}, \mathrm{dd}, J=$ 12.3, 3.1 Hz, H-6'a), 4.60 (1H, dd, $J=12.3,5.1 \mathrm{~Hz}, \mathrm{H}-6$ 'b), 4.39 (1H, ddd, $J=9.7,4.9,3.2$ Hz, H-5'), $4.10\left(1 \mathrm{H}, \mathrm{d}, J=15.8 \mathrm{~Hz}, \mathrm{PhCH}_{2}\right), 4.19\left(1 \mathrm{H}, \mathrm{d}, J=15.8 \mathrm{~Hz}, \mathrm{PhCH}_{2}\right) ;{ }^{13} \mathrm{C} \mathrm{NMR}$ $\left(100 \mathrm{MHz}, \mathrm{CDCl}_{3}\right) \delta(\mathrm{ppm}): 166.3,166.0,165.3,164.7(\mathrm{C}=\mathrm{O}), 158.8,155.4$ (triazole C-3, C5), 136.9, 135.5 (q, Ph), 133.4-125.1 (aromatics), 76.9, 74.7, 74.5, 71.7, 69.9 (C-1'-C-5'), $63.7\left(\mathrm{C}-6\right.$ '), $32.5\left(\mathrm{PhCH}_{2}\right)$. ESI-MS positive mode (m/z): calcd for $\mathrm{C}_{49} \mathrm{H}_{40} \mathrm{~N}_{3} \mathrm{O}_{9}\left([\mathrm{M}+\mathrm{H}]^{+}\right)$: 814.276. Found: 814.28. Anal. Calcd for $\mathrm{C}_{49} \mathrm{H}_{39} \mathrm{~N}_{3} \mathrm{O}_{9}$ : C, 72.31; H, 4.83; N, 5.16. Found: C, 70.57; H, 4.98; N, 5.10.

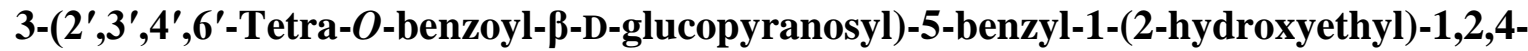

\section{triazole (32)}

Prepared from compound $15(0.31 \mathrm{~g}, 0.41 \mathrm{mmol})$ and 2-hydroxyethylhydrazine (33 $\mu \mathrm{L}, 0.49$ mmol) according to general procedure IV. Purified by column chromatography (1:3 EtOAc/hexane) to give $0.23 \mathrm{~g}(72 \%)$ pale yellow amorphous solid. $\mathbf{R}_{\mathrm{f}}=0.42(1: 1$ 
Acetone/hexane); $[\alpha]_{\mathrm{D}}=+16\left(\mathrm{c} 0.53, \mathrm{CHCl}_{3}\right) ;{ }^{1} \mathrm{H} \mathrm{NMR}\left(400 \mathrm{MHz}, \mathrm{CDCl}_{3}\right) \delta(\mathrm{ppm}): 8.00(2 \mathrm{H}$, $\mathrm{d}, J=7.5 \mathrm{~Hz}$, aromatics), $7.93(2 \mathrm{H}, \mathrm{d}, J=7.4 \mathrm{~Hz}$, aromatics), 7.86-7.81 (4H, m, aromatics), 7.50-7.23 (12H, m, aromatics), 7.14-7.13 (3H, m, aromatics), 7.02-6.99 (2H, m, aromatics), 6.10-6.06 (2H, m, H-2' and/or H-3' and/or H-4'), 5.87 (1H, pseudo t, $J=9.5 \mathrm{~Hz}, \mathrm{H}-2$ ' or H3' or H-4'), 5.04 (1H, d, $J=9.7$ Hz, H-1'), 4.65 (1H, dd, $J=12.2,2.8$ Hz, H-6'a), 4.57 (1H, $\left.\mathrm{dd}, J=12.2,5.2 \mathrm{~Hz}, \mathrm{H}-6^{\prime} \mathrm{b}\right), 4.36(1 \mathrm{H}, \mathrm{ddd}, J=9.6,5.0,3.1 \mathrm{~Hz}, \mathrm{H}-5$ '), $4.11(2 \mathrm{H}, \mathrm{q}, J=16.1$ $\left.\mathrm{Hz}, \mathrm{PhCH}_{2}\right), 3.94-3.89\left(2 \mathrm{H}, \mathrm{m}, \mathrm{CH}_{2}\right), 3.84-3.80\left(1 \mathrm{H}, \mathrm{m}, \mathrm{CH}_{2}\right), 3.66-3.62\left(1 \mathrm{H}, \mathrm{m}, \mathrm{CH}_{2}\right) ;{ }^{13} \mathrm{C}$ NMR (100 MHz, $\left.\mathrm{CDCl}_{3}\right) \delta(\mathrm{ppm}): 166.3,165.9,165.3,165.3(\mathrm{C}=\mathrm{O}), 158.2,155.8$ (triazole C-3, C-5), 135.0 (q, Ph), 133.4-127.1 (aromatics), 76.8, 74.6, 74.2, 72.3, 69.8 (C-1'-C-5'), $63.7\left(\mathrm{C}-6\right.$ ') $, 60.5,50.7\left(2 \times \mathrm{CH}_{2}\right), 32.0\left(\mathrm{PhCH}_{2}\right)$. ESI-MS positive mode $(\mathrm{m} / \mathrm{z})$ : calcd for $\mathrm{C}_{45} \mathrm{H}_{40} \mathrm{~N}_{3} \mathrm{O}_{10}\left([\mathrm{M}+\mathrm{H}]^{+}\right):$782.271. Found: 782.27. Anal. Calcd for $\mathrm{C}_{45} \mathrm{H}_{39} \mathrm{~N}_{3} \mathrm{O}_{10}: \mathrm{C}, 69.13 ; \mathrm{H}$, 5.03; N, 5.37. Found: C, 69.46; H, 5.17; N, 5.28.

\section{3-(ק-D-Glucopyranosyl)-5-methyl-1-phenyl-1,2,4-triazole (33)}

From triazole $21(0.27 \mathrm{~g}, 0.36 \mathrm{mmol})$ according to general procedure V. Purified by column chromatography $\left(3: 1 \mathrm{CHCl}_{3} / \mathrm{MeOH}\right)$ to yield $0.08 \mathrm{~g}(70 \%)$ pale yellow syrup. $\mathrm{R}_{\mathrm{f}}=0.54(7: 3$ $\left.\mathrm{CHCl}_{3} / \mathrm{MeOH}\right) ;[\alpha]_{\mathrm{D}}=+16(\mathrm{c} 0.51, \mathrm{MeOH}) ; 1 \mathrm{H} \mathrm{NMR}\left(400 \mathrm{MHz}, \mathrm{CD}_{3} \mathrm{OD}\right) \delta(\mathrm{ppm}): 7.60$ $7.52\left(5 \mathrm{H}, \mathrm{m}\right.$, aromatics), $4.37\left(1 \mathrm{H}, \mathrm{d}, J=9.7 \mathrm{~Hz}, \mathrm{H}-1^{\prime}\right), 3.88(1 \mathrm{H}, \mathrm{dd}, J=12.1,1.9 \mathrm{~Hz}, \mathrm{H}-$ 6'a), $3.78(1 \mathrm{H}$, pseudo t, $J=9.2 \mathrm{~Hz}, \mathrm{H}-2$ ' or H-3' or H-4'), $3.71(1 \mathrm{H}, \mathrm{dd}, J=12.1,5.0 \mathrm{~Hz}, \mathrm{H}-$ 6'b), $3.53(1 \mathrm{H}$, pseudo t, $J=8.7 \mathrm{~Hz}, \mathrm{H}-2$ ' or H-3' or H-4'), $3.49(1 \mathrm{H}$, pseudo t, $J=8.9 \mathrm{~Hz}, \mathrm{H}-$ 2' or H-3' or H-4'), 3.46-3.42 (1H, m, H-5'), $2.50\left(3 \mathrm{H}, \mathrm{s}, \mathrm{CH}_{3}\right) ;{ }^{13} \mathrm{C} \mathrm{NMR}(100 \mathrm{MHz}$, $\left.\mathrm{CD}_{3} \mathrm{OD}\right) \delta(\mathrm{ppm}): 161.8,154.7$ (triazole C-3, C-5), 138.4 (q, Ph), 130.7, 130.4, 126.0 (aromatics), 82.3, 79.3, 76.8, 74.3, $71.3\left(\mathrm{C}-1^{\prime}-\mathrm{C}-5\right.$ '), $62.9\left(\mathrm{C}-6\right.$ ') $12.9\left(\mathrm{CH}_{3}\right)$. ESI-MS positive mode $(\mathrm{m} / \mathrm{z})$ : calcd for $\mathrm{C}_{15} \mathrm{H}_{20} \mathrm{~N}_{3} \mathrm{O}_{5}\left([\mathrm{M}+\mathrm{H}]^{+}\right)$: 322.140. Found: 322.33. Anal. Calcd for $\mathrm{C}_{15} \mathrm{H}_{19} \mathrm{~N}_{3} \mathrm{O}_{5}$ : C, 56.07; H, 5.96; N, 13.08. Found: C, 56.20; H, 6.09; N, 13.13. 


\section{3-(ß-D-Glucopyranosyl)-1-(2-hydroxyethyl)-5-methyl-1,2,4-triazole (34)}

From triazole $22(0.24 \mathrm{~g}, 0.34 \mathrm{mmol})$ according to general procedure V. Purified by column chromatography $\left(7: 3 \mathrm{CHCl}_{3} / \mathrm{MeOH}\right)$ to yield $0.07 \mathrm{~g}(72 \%)$ colourless syrup. $\mathrm{R}_{\mathrm{f}}=0.21(7: 3$ $\left.\mathrm{CHCl}_{3} / \mathrm{MeOH}\right) ;[\alpha]_{\mathrm{D}}=+16(\mathrm{c} 0.73, \mathrm{MeOH}) ; 1 \mathrm{H} \mathrm{NMR}\left(400 \mathrm{MHz}, \mathrm{CD}_{3} \mathrm{OD}\right) \delta(\mathrm{ppm}): 4.28$ $\left(1 \mathrm{H}, \mathrm{d}, J=9.7 \mathrm{~Hz}, \mathrm{H}-1^{\prime}\right), 4.19\left(2 \mathrm{H}, \mathrm{t}, J=5.1 \mathrm{~Hz}, \mathrm{CH}_{2}\right), 3.90\left(2 \mathrm{H}, \mathrm{t}, J=5.1 \mathrm{~Hz}, \mathrm{CH}_{2}\right), 3.86$ (1H, dd, $J=12.1,1.9$ Hz, H-6'a), 3.74-3.65 (2H, m, H-2' or H-3' or H-4' and H-6'b), 3.533.45 (2H, m, H-2' and/or H-3' and/or H-4'), 3.44-3.40 (1H, m, H-5'), $2.48\left(3 \mathrm{H}, \mathrm{s}, \mathrm{CH}_{3}\right) ;{ }^{13} \mathrm{C}$ NMR (100 MHz, CD 3 OD) $\delta$ (ppm): 161.0, 155.5 (triazole C-3, C-5), 82.2, 79.2, 76.8, 74.3, $71.3\left(\mathrm{C}-1\right.$ '-C-5'), 62.9 (C-6'), 61.3, $51.9\left(2 \times \mathrm{CH}_{2}\right), 11.8\left(\mathrm{CH}_{3}\right)$. ESI-MS positive mode $(\mathrm{m} / \mathrm{z})$ : calcd for $\mathrm{C}_{11} \mathrm{H}_{20} \mathrm{~N}_{3} \mathrm{O}_{6}\left([\mathrm{M}+\mathrm{H}]^{+}\right)$: 290.135. Found: 290.33. Anal. Calcd for $\mathrm{C}_{11} \mathrm{H}_{19} \mathrm{~N}_{3} \mathrm{O}_{6}$ : C, 45.67; H, 6.62; N, 14.53. Found: C, 45.78; H, 6.70; N, 14.65.

\section{3-(ק-D-Glucopyranosyl)-5-hydroxymethyl-1-phenyl-1,2,4-triazole (35)}

From triazole $25(0.21 \mathrm{~g}, 0.27 \mathrm{mmol})$ according to general procedure V. Purified by column chromatography (4:1 $\left.\mathrm{CHCl}_{3} / \mathrm{MeOH}\right)$ to yield $0.08 \mathrm{~g}(84 \%)$ pale yellow syrup. $\mathrm{R}_{\mathrm{f}}=0.35$ (3:1 $\left.\mathrm{CHCl}_{3} / \mathrm{MeOH}\right) ;[\alpha]_{\mathrm{D}}=+18(\mathrm{c} 0.54, \mathrm{MeOH}) ; 1 \mathrm{H} \mathrm{NMR}\left(400 \mathrm{MHz}, \mathrm{CD}_{3} \mathrm{OD}\right) \delta(\mathrm{ppm}): 7.66-$ $7.63\left(2 \mathrm{H}, \mathrm{m}\right.$, aromatics), 7.60-7.50 (3H, m, aromatics), $4.67\left(2 \mathrm{H}, \mathrm{s}, \mathrm{CH}_{2}\right), 4.44(1 \mathrm{H}, \mathrm{d}, J=9.7$ Hz, H-1'), 3.88 (1H, dd, $J=12.2,1.8 \mathrm{~Hz}, \mathrm{H}-6$ 'a), 3.82 (1H, pseudo t, $J=9.2 \mathrm{~Hz}, \mathrm{H}-2$ ' or H-3' or H-4'), 3.71 (1H, dd, $J=12.1,4.9 \mathrm{~Hz}, \mathrm{H}-6$ 'b), 3.63-3.45 (3H, m, H-2' and/or H-3' and/or H-4', H-5'); ${ }^{13} \mathrm{C}$ NMR (100 MHz, CD $\left.{ }_{3} \mathrm{OD}\right) \delta(\mathrm{ppm}): 162.0,156.6$ (triazole C-3, C-5), 138.2 (q, Ph), 130.5, 130.4, 125.7 (aromatics), 81.2, 79.3, 76.8, 74.2, 71.2 (C-1'-C-5'), 62.8 (C-6'), 55.6 $\left(\mathrm{CH}_{2}\right)$. ESI-MS positive mode $(\mathrm{m} / \mathrm{z})$ : calcd for $\mathrm{C}_{15} \mathrm{H}_{20} \mathrm{~N}_{3} \mathrm{O}_{6}\left([\mathrm{M}+\mathrm{H}]^{+}\right)$: 338.340. Found: 338.42. Anal. Calcd for $\mathrm{C}_{15} \mathrm{H}_{19} \mathrm{~N}_{3} \mathrm{O}_{6}$ : C, 53.41; H, 5.68; N, 12.46. Found: C, 53.49; H, 5.77; $\mathrm{N}, 12.52$. 


\section{3-(ß-D-Glucopyranosyl)-1-(2-hydroxyethyl)-5-hydroxymethyl-1,2,4-triazole (36)}

From triazole 26 ( $0.17 \mathrm{~g}, 0.24 \mathrm{mmol})$ according to general procedure V. Purified by column chromatography $\left(4: 1 \mathrm{CHCl}_{3} / \mathrm{MeOH}\right)$ to yield $0.04 \mathrm{~g}(62 \%)$ colourless syrup. $\mathrm{R}_{\mathrm{f}}=0.29(7: 3$ $\left.\mathrm{CHCl}_{3} / \mathrm{MeOH}\right) ;[\alpha]_{\mathrm{D}}=+13(\mathrm{c} 0.53, \mathrm{MeOH}) ; 1 \mathrm{H} \mathrm{NMR}\left(360 \mathrm{MHz}, \mathrm{CD}_{3} \mathrm{OD}\right) \delta(\mathrm{ppm}): 4.76$ $\left(2 \mathrm{H}, \mathrm{s}, \mathrm{CH}_{2}\right), 4.36\left(2 \mathrm{H}, \mathrm{t}, J=5.3 \mathrm{~Hz}, \mathrm{CH}_{2}\right), 4.31\left(1 \mathrm{H}, \mathrm{d}, J=9.7 \mathrm{~Hz}, \mathrm{H}-1^{\prime}\right), 3.91(2 \mathrm{H}, \mathrm{t}, J=5.3$ Hz, $\left.\mathrm{CH}_{2}\right), 3.84$ (1H, dd, $J=12.1,1.9$ Hz, H-6'a), 3.74-3.64 (2H, m, H-2' or H-3' or H-4', H6'b), 3.52-3.38 (3H, m, H-2' and/or H-3' and/or H-4', H-5'); ${ }^{13} \mathrm{C}$ NMR (90 MHz, CD $\left.3 \mathrm{OD}\right) \delta$ (ppm): 161.3, 157.5 (triazole C-3, C-5), 82.2, 79.3, 76.9, 74.3, 71.4 (C-1'-C-5'), 62.9 (C-6'), 61.3, 55.9, $52.3\left(3 \times \mathrm{CH}_{2}\right)$. ESI-MS positive mode $(\mathrm{m} / \mathrm{z})$ : calcd for $\mathrm{C}_{11} \mathrm{H}_{20} \mathrm{~N}_{3} \mathrm{O}_{7}\left([\mathrm{M}+\mathrm{H}]^{+}\right)$: 306.295. Found: 306.33. Anal. Calcd for $\mathrm{C}_{11} \mathrm{H}_{19} \mathrm{~N}_{3} \mathrm{O}_{7}$ : C, 43.28, H, 6.27; N, 13.76. Found: C, 43.41; H, 6.39; N, 13.71 .

\section{5-(tert-Butyl)-3-( $\beta$-D-glucopyranosyl)-1-phenyl-1,2,4-triazole (37)}

From triazole $28(0.13 \mathrm{~g}, 0.17 \mathrm{mmol})$ according to general procedure V. Purified by column chromatography $\left(6: 1 \mathrm{CHCl}_{3} / \mathrm{MeOH}\right)$ to yield $0.03 \mathrm{~g}(50 \%)$ colourless syrup. $\mathrm{R}_{\mathrm{f}}=0.32(5: 1$ $\left.\mathrm{CHCl}_{3} / \mathrm{MeOH}\right) ;[\alpha]_{\mathrm{D}}=+8(\mathrm{c} 0.52, \mathrm{MeOH}) ; 1 \mathrm{H} \mathrm{NMR}\left(360 \mathrm{MHz}, \mathrm{CD}_{3} \mathrm{OD}\right) \delta(\mathrm{ppm}): 7.61-7.54$ (3H, m, aromatics), 7.49-7.44 (2H, m, aromatics), $4.34(1 \mathrm{H}, \mathrm{d}, J=9.7 \mathrm{~Hz}, \mathrm{H}-1$ '), 4.20 (2H, s, $\left.\mathrm{PhCH}_{2}\right), 3.86$ (1H, dd, $J=12.1,1.8 \mathrm{~Hz}, \mathrm{H}-6$ 'a), 3.78 (1H, pseudo t, $J=9.2 \mathrm{~Hz}, \mathrm{H}-2$ ' or H-3' or H-4'), 3.69 (1H, dd, $J=12.1,4.9 \mathrm{~Hz}, \mathrm{H}-6$ 'b), 3.54-3.40 (3H, m, H-2' and/or H-3' and/or H-4', H-5'), 1.27 (9H, s, C(CH$\left.\left.)_{3}\right) ;{ }^{13} \mathrm{C} \mathrm{NMR} \mathrm{(90} \mathrm{MHz,} \mathrm{CD}_{3} \mathrm{OD}\right) \delta(\mathrm{ppm}): 165.4,160.8$ (triazole C-3, C-5), 140.7 (q, Ph), 131.4, 130.3, 129.3 (aromatics), 82.3, 79.4, 76.9, 74.2, 71.3 (C-1'-C-5'), $62.9\left(\mathrm{C}-6\right.$ '), $34.7\left(\mathrm{C}\left(\mathrm{CH}_{3}\right)_{3}\right), 30.3\left(\mathrm{C}\left(\mathrm{CH}_{3}\right)_{3}\right)$. ESI-MS positive mode (m/z): calcd for $\mathrm{C}_{18} \mathrm{H}_{26} \mathrm{~N}_{3} \mathrm{O}_{5}\left([\mathrm{M}+\mathrm{H}]^{+}\right)$: 364.423. Found: 364.42. Anal. Calcd for $\mathrm{C}_{18} \mathrm{H}_{25} \mathrm{~N}_{3} \mathrm{O}_{5}: \mathrm{C}, 59.49 ; \mathrm{H}$, 6.93; N, 11.56. Found: C, 59.57; H, 6.90; N, 11.70. 


\section{5-(tert-Butyl)-3-( $\beta$-D-glucopyranosyl)-1-(2-hydroxyethyl)-1,2,4-triazole (38)}

From triazole $29(0.22 \mathrm{~g}, 0.29 \mathrm{mmol})$ according to general procedure V. Purified by column chromatography $\left(5: 1 \mathrm{CHCl}_{3} / \mathrm{MeOH}\right)$ to yield $0.07 \mathrm{~g}(65 \%)$ white amorphous solid. $\mathrm{R}_{\mathrm{f}}=0.25$ $\left(5: 1 \mathrm{CHCl}_{3} / \mathrm{MeOH}\right) ;[\alpha]_{\mathrm{D}}=+10(\mathrm{c} 0.59, \mathrm{MeOH}) ; 1 \mathrm{H} \mathrm{NMR}\left(400 \mathrm{MHz}, \mathrm{CD}_{3} \mathrm{OD}\right) \delta(\mathrm{ppm}) ;$ $4.36\left(2 \mathrm{H}, \mathrm{t}, J=5.6 \mathrm{~Hz}, \mathrm{CH}_{2}\right), 4.28(1 \mathrm{H}, \mathrm{d}, J=9.8 \mathrm{~Hz}, \mathrm{H}-1$ ' $), 4.00\left(2 \mathrm{H}, \mathrm{t}, J=5.6 \mathrm{~Hz}, \mathrm{CH}_{2}\right)$, $3.84(1 \mathrm{H}, \mathrm{dd}, J=12.0,1.9 \mathrm{~Hz}, \mathrm{H}-6$ 'a), 3.75 (1H, pseudo t, $J=9.2 \mathrm{~Hz}, \mathrm{H}-2$ ' or H-3' or H-4'), 3.67 (1H, dd, $J=12.0,5.0 \mathrm{~Hz}, \mathrm{H}-6$ 'b), 3.50-3.36 (3H, m, H-2' and/or H-3' and/or H-4', H5'), 1.47 (9H, s, $\left.\mathrm{C}\left(\mathrm{CH}_{3}\right)_{3}\right)$ ); ${ }^{13} \mathrm{C} \mathrm{NMR}\left(100 \mathrm{MHz}, \mathrm{CD}_{3} \mathrm{OD}\right) \delta(\mathrm{ppm}): 164.9,160.3$ (triazole C3, C-5), 82.1, 79.2, 76.9, 74.1, 71.3 (C-1'-C-5'), 62.8 (C-6'), 61.3, $53.5\left(2 \times \mathrm{CH}_{2}\right), 33.8$ $\left(C\left(\mathrm{CH}_{3}\right)_{3}\right), 29.8\left(\mathrm{C}\left(\mathrm{CH}_{3}\right)_{3}\right)$. ESI-MS positive mode $(\mathrm{m} / \mathrm{z})$ : calcd for $\mathrm{C}_{14} \mathrm{H}_{26} \mathrm{~N}_{3} \mathrm{O}_{6}\left([\mathrm{M}+\mathrm{H}]^{+}\right)$: 332.377. Found: 332.42. Anal. Calcd for $\mathrm{C}_{14} \mathrm{H}_{25} \mathrm{~N}_{3} \mathrm{O}_{6}$ : C, 50.75; H, 7.60; N, 12.68. Found: C, 50.70; H, 7.53; N, 12.72 .

\section{5-Benzyl-3-( $\beta$-D-glucopyranosyl)-1,2,4-triazole (39)}

From triazole $30(0.18 \mathrm{~g}, 0.24 \mathrm{mmol})$ according to general procedure $\mathrm{V}$. (The mixture was neutralised with acetic acid.) Purified by column chromatography $\left(5: 1 \mathrm{CHCl}_{3} / \mathrm{MeOH}\right)$ to yield $0.03 \mathrm{~g}(45 \%)$ colourless syrup. $\mathrm{R}_{\mathrm{f}}=0.34\left(7: 3 \mathrm{CHCl}_{3} / \mathrm{MeOH}\right) ;[\alpha]_{\mathrm{D}}=+10(\mathrm{c} 0.53, \mathrm{MeOH}) ; 1 \mathrm{H}$

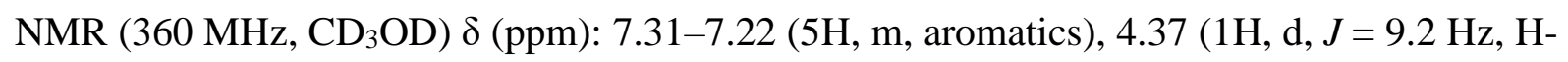
1'), 4.10 (2H, s, $\left.\mathrm{PhCH}_{2}\right), 3.85$ (1H, dd, J=12.0, $1.4 \mathrm{~Hz}, \mathrm{H}-6$ 'a), 3.68 (2H, H-2' or H-3' or H4' or H-5', H-6'b), 3.51-3.38 (3H, m, H-2' and/or H-3' and/or H-4' and/or H-5'); ${ }^{13} \mathrm{C} \mathrm{NMR}$ $\left(90 \mathrm{MHz}, \mathrm{CD}_{3} \mathrm{OD}\right) \delta(\mathrm{ppm}): 162.9,157.7$ (triazole C-3, C-5), 137.6 (q, Ph), 129.7, 128.0 (aromatics), 82.2, 79.3, 76.8, 74.3, 71.3 (C-1'-C-5'), 62.8 (C-6'), 33.5 ( $\left.\mathrm{PhCH}_{2}\right)$. ESI-MS positive mode $(\mathrm{m} / \mathrm{z})$ : calcd for $\mathrm{C}_{15} \mathrm{H}_{20} \mathrm{~N}_{3} \mathrm{O}_{5}\left([\mathrm{M}+\mathrm{H}]^{+}\right)$: 322.140. Found: 322.33. Anal. Calcd for $\mathrm{C}_{15} \mathrm{H}_{19} \mathrm{~N}_{3} \mathrm{O}_{5}$ : C, 56.07; H, 5.96; N, 13.08. Found: C, 56.14; H, 5.99; N, 13.25. 


\section{5-Benzyl-3-( $\beta$-D-glucopyranosyl)-1-phenyl-1,2,4-triazole (40)}

From triazole 31 ( $0.170 \mathrm{~g}, 0.21 \mathrm{mmol})$ according to general procedure V. Purified by column chromatography $\left(5: 1 \mathrm{CHCl}_{3} / \mathrm{MeOH}\right)$ to yield $0.06 \mathrm{~g}(70 \%)$ pale yellow syrup. $\mathrm{R}_{\mathrm{f}}=0.55(7: 3$ $\left.\mathrm{CHCl}_{3} / \mathrm{MeOH}\right) ;[\alpha]_{\mathrm{D}}=+11(\mathrm{c} 0.53, \mathrm{MeOH}) ; 1 \mathrm{H} \mathrm{NMR}\left(400 \mathrm{MHz}, \mathrm{CD}_{3} \mathrm{OD}\right) \delta(\mathrm{ppm}): 7.52-$ $7.49(3 \mathrm{H}, \mathrm{m}$, aromatics), 7.52-7.49 (3H, m, aromatics), 7.25-7.18 (3H, m, aromatics), 7.07$7.03\left(2 \mathrm{H}, \mathrm{m}\right.$, aromatics), $4.42\left(1 \mathrm{H}, \mathrm{d}, J=9.7 \mathrm{~Hz}, \mathrm{H}-1^{\prime}\right), 4.19\left(2 \mathrm{H}, \mathrm{s}, \mathrm{PhCH}_{2}\right), 3.89(1 \mathrm{H}, \mathrm{dd}, J$ = 12.1, $1.8 \mathrm{~Hz}, \mathrm{H}-6$ 'a), 3.83 (1H, pseudo t, $J=9.2 \mathrm{~Hz}, \mathrm{H}-2^{\prime}$ ' or H-3' or H-4'), 3.72 (1H, dd, $J$ $=12.1,5.0 \mathrm{~Hz}, \mathrm{H}-6$ 'b), 3.58-3.45 (3H, m, H-2' and/or H-3' and/or H-4', H-5'); ${ }^{13} \mathrm{C} \mathrm{NMR}$ (100 MHz, CD $\left.{ }_{3} \mathrm{OD}\right) \delta(\mathrm{ppm}): 162.1,156.8$ (triazole C-3, C-5), 138.2, 136.9 (q, Ph), 130.7, 130.6, 129.7, 129.5, 128.1, 126.7 (aromatics), 82.3, 79.4, 76.9, 74.3, 71.3 (C-1'-C-5'), 62.9 (C-6'), $33.1\left(\mathrm{PhCH}_{2}\right)$. ESI-MS positive mode (m/z): calcd for $\mathrm{C}_{21} \mathrm{H}_{24} \mathrm{~N}_{3} \mathrm{O}_{5}\left([\mathrm{M}+\mathrm{H}]^{+}\right)$: 398.171. Found: 398.42. Anal. Calcd for $\mathrm{C}_{21} \mathrm{H}_{23} \mathrm{~N}_{3} \mathrm{O}_{5}$ : C, 63.47; H, 5.83; N, 10.57. Found: C, 63.69; H, 5.90; N, 10.56 .

\section{5-Benzyl-3-( $\beta$-D-glucopyranosyl)-1-(2-hydroxyethyl)-1,2,4-triazole (41)}

From triazole $32(0.23 \mathrm{~g}, 0.29 \mathrm{mmol})$ according to general procedure V. Purified by column chromatography $\left(5: 1 \mathrm{CHCl}_{3} / \mathrm{MeOH}\right)$ to yield $0.06 \mathrm{~g}(51 \%)$ colourless syrup. $\mathrm{R}_{\mathrm{f}}=0.34(7: 3$ $\left.\mathrm{CHCl}_{3} / \mathrm{MeOH}\right) ;[\alpha]_{\mathrm{D}}=+6(\mathrm{c} 0.53, \mathrm{MeOH}) ; 1 \mathrm{H} \mathrm{NMR}\left(400 \mathrm{MHz}, \mathrm{CD}_{3} \mathrm{OD}\right) \delta(\mathrm{ppm}): 7.35-7.23$ (5H, m, aromatics), $4.32\left(1 \mathrm{H}, \mathrm{d}, J=9.7 \mathrm{~Hz}, \mathrm{H}-1^{\prime}\right), 4.26\left(2 \mathrm{H}, \mathrm{s}, \mathrm{PhCH}_{2}\right), 4.16(2 \mathrm{H}, \mathrm{t}, J=5.3$ $\left.\mathrm{Hz}, \mathrm{CH}_{2}\right), 3.87\left(1 \mathrm{H}, \mathrm{dd}, J=12.3,2.0 \mathrm{~Hz}, \mathrm{H}-6\right.$ 'a), $3.83\left(2 \mathrm{H}, \mathrm{t}, J=5.3 \mathrm{~Hz}, \mathrm{CH}_{2}\right), 3.75(1 \mathrm{H}$, pseudo t, $J=9.2 \mathrm{~Hz}, \mathrm{H}-2$ ' or H-3' or H-4'), 3.69 (1H, dd, $J=12.1,5.0 \mathrm{~Hz}, \mathrm{H}-6$ 'b), 3.54-3.44 (2H, m, H-2' and/or H-3' and/or H-4'), 3.44-3.40 (1H, m, H-5'); ${ }^{13} \mathrm{C}$ NMR (100 MHz, $\left.\mathrm{CD}_{3} \mathrm{OD}\right) \delta(\mathrm{ppm}): 161.5,157.4$ (triazole C-3, C-5), 137.0 (q, Ph), 129.9, 129.7, 128.1 (aromatics), 82.3, 79.3, 76.9, 74.3, $71.3\left(\mathrm{C}-1^{\prime}{ }^{-}-\mathrm{C}-5\right.$ ') $), 62.9$ (C-6'), 61.1, $52.0\left(2 \times \mathrm{CH}_{2}\right), 32.6$ 
$\left(\mathrm{PhCH}_{2}\right)$. ESI-MS positive mode $(\mathrm{m} / \mathrm{z})$ : calcd for $\mathrm{C}_{17} \mathrm{H}_{24} \mathrm{~N}_{3} \mathrm{O}_{6}\left([\mathrm{M}+\mathrm{H}]^{+}\right): 366.166$. Found: 366.33. Anal. Calcd for $\mathrm{C}_{17} \mathrm{H}_{23} \mathrm{~N}_{3} \mathrm{O}_{6}$ : C, 55.88, H, 6.35; N, 11.50. Found: C, 56.00; H, 6.42; N, 11.44 .

\section{5-(2',3',4',6'-Tetra-O-benzoyl- $\beta$-D-glucopyranosyl)-3-methyl-1-phenyl-1,2,4-triazole (42)}

Prepared from compound $18(0.14 \mathrm{~g}, 0.21 \mathrm{mmol})$ and phenylhydrazine $(25 \mu \mathrm{L}, 0.25 \mathrm{mmol})$ according to general procedure IV. Purified by column chromatography (1:3 EtOAc/hexane) to give $0.10 \mathrm{~g}(65 \%)$ yellow amorphous solid. $\mathrm{R}_{\mathrm{f}}=0.29(1: 2 \mathrm{EtOAc} / \mathrm{hexane}) ;[\alpha]_{\mathrm{D}}=+47(\mathrm{c}$ 0.345, $\left.\mathrm{CHCl}_{3}\right) ;{ }^{1} \mathrm{H} \mathrm{NMR}\left(360 \mathrm{MHz}, \mathrm{CDCl}_{3}\right) \delta(\mathrm{ppm}): 8.05(2 \mathrm{H}, \mathrm{dd}, J=8.2,1.3 \mathrm{~Hz}$, aromatics), $7.91(2 \mathrm{H}, \mathrm{dd}, J=8.2,1.4 \mathrm{~Hz}$, aromatics), $7.82(2 \mathrm{H}, \mathrm{dd}, J=8.2,1.4 \mathrm{~Hz}$, aromatics), 7.73 (2H, dd, $J=8.2,1.3$, aromatics), 7.88-7.80 (4H, m, aromatics), 7.59-7.25 (17H, m, aromatics), 6.21 (pseudo t, $1 \mathrm{H}, J=9.7 \mathrm{~Hz}, \mathrm{H}-2$ ' or $\mathrm{H}-3$ ' or $\mathrm{H}-4$ '), 5.91 (1H, pseudo t, $J=9.5 \mathrm{~Hz}, \mathrm{H}-2^{\prime}$ or H-3' or H-4'), 5.70 (1H, pseudo t, $J=9.5 \mathrm{~Hz}, \mathrm{H}-2^{\prime}$ ' or H-3' or H-4'), $4.96\left(1 \mathrm{H}, \mathrm{d}, J=9.9 \mathrm{~Hz}, \mathrm{H}-1^{\prime}\right), 4.70$ (1H, dd, $J=12.3,2.7 \mathrm{~Hz}, \mathrm{H}-6$ 'a), 4.46 (1H, dd, $J=12.3$, 6.4 Hz, H-6'b), 4.27 (1H, ddd, $J=12.3,6.4,2.7 \mathrm{~Hz}, \mathrm{H}-5$ ') $2.37\left(3 \mathrm{H}, \mathrm{s}, \mathrm{CH}_{3}\right) ;{ }^{13} \mathrm{C} \mathrm{NMR}(90$ $\left.\mathrm{MHz}, \mathrm{CDCl}_{3}\right) \delta(\mathrm{ppm}): 166.2,165.9,165.3,164.3(\mathrm{C}=\mathrm{O}), 161.1,150.1$ (triazole C-3, C-5), 136.9-125.1 (aromatics), 77.0, 74.3, 71.5, 71.0, 69.6 (C-1'-C-5'), 63.6 (C-6'), $13.9\left(\mathrm{CH}_{3}\right)$. ESI-MS positive mode (m/z): calcd for $\mathrm{C}_{43} \mathrm{H}_{36} \mathrm{~N}_{3} \mathrm{O}_{9}\left([\mathrm{M}+\mathrm{H}]^{+}\right)$: 738.245. Found: 738.29. Anal. Calcd for $\mathrm{C}_{43} \mathrm{H}_{35} \mathrm{~N}_{3} \mathrm{O}_{9}$ : C, 70.01; H, 4.78; N, 5.70. Found: C, 70.19; H, 4.90; N, 5.63.

\section{3-(2',3',4',6'-Tetra- $O$-benzoyl- $\beta$-D-glucopyranosyl)-5-phenyl-1,2,4-triazole (43)}

Prepared from compound $19(0.10 \mathrm{~g}, 0.13 \mathrm{mmol})$ hydrazinium acetate $(14 \mathrm{mg}, 0.16 \mathrm{mmol})$ according to general procedure IV. Purification by column chromatography (1:2 EtOAc/hexane) yielded $0.03 \mathrm{~g}(35 \%)$ white solid. The compound characterization data are identical with those reported ${ }^{20}$. 


\section{5-(2',3',4',6'-Tetra- $O$-benzoyl- $\beta$-D-glucopyranosyl)-1,3-diphenyl-1,2,4-triazole (44)}

Prepared from compound $19(0.15 \mathrm{~g}, 0.20 \mathrm{mmol})$ and phenylhydrazine $(24 \mu \mathrm{L}, 0.24 \mathrm{mmol})$ according to general procedure IV. Purified by column chromatography (1:3 EtOAc/hexane) to give $0.14 \mathrm{~g}(90 \%)$ yellow amorphous solid. $\mathrm{R}_{\mathrm{f}}=0.49(1: 2 \mathrm{EtOAc} / \mathrm{hexane}) ;[\alpha]_{\mathrm{D}}=+19(\mathrm{c}$ 0.38, $\left.\mathrm{CHCl}_{3}\right) ;{ }^{1} \mathrm{H} \mathrm{NMR}\left(360 \mathrm{MHz}, \mathrm{CDCl}_{3}\right) \delta$ (ppm): 8.09-8.05 (4H, m, aromatics), $7.93(2 \mathrm{H}$, $\mathrm{d}, J=7.5 \mathrm{~Hz}$, aromatics), $7.85(2 \mathrm{H}, \mathrm{d}, J=7.5 \mathrm{~Hz}$, aromatics $), 7.72(2 \mathrm{H}, \mathrm{d}, J=7.5 \mathrm{~Hz}$, aromatics), 7.64 (2H, d, $J=7.5 \mathrm{~Hz}$, aromatics), 7.59-7.21 (18H, m, aromatics), 6.42 (pseudo t, $1 \mathrm{H}, J=9.7 \mathrm{~Hz}, \mathrm{H}-2^{\prime}$ or H-3' or H-4'), 5.95 (1H, pseudo t, $J=9.5 \mathrm{~Hz}, \mathrm{H}-2^{\prime}$ or H-3' or H4'), $5.73(1 \mathrm{H}$, pseudo t, $J=9.7 \mathrm{~Hz}, \mathrm{H}-2$ ' or $\mathrm{H}-3$ ' or H-4'), $5.00(1 \mathrm{H}, \mathrm{d}, J=9.9 \mathrm{~Hz}, \mathrm{H}-1$ ' $), 4.72$ $(1 \mathrm{H}, \mathrm{dd}, J=12.2,2.0 \mathrm{~Hz}, \mathrm{H}-6$ 'a), 4.46 (1H, dd, $J=12.2,6.6 \mathrm{~Hz}, \mathrm{H}-6$ 'b), 4.30 (1H, ddd, $J=$ 12.2, 6.6, $2.0 \mathrm{~Hz}, \mathrm{H}-5$ '); ${ }^{13} \mathrm{C} \mathrm{NMR}\left(90 \mathrm{MHz}, \mathrm{CDCl}_{3}\right) \delta$ (ppm): 166.2, 166.0, 165.4, 164.5 $(\mathrm{C}=\mathrm{O}), 162.1,150.6$ (triazole C-3, C-5), 137.1-125.1 (aromatics), 77.1, 74.4, 71.7, 70.7, 69.7 (C-1'-C-5'), 63.7 (C-6'). ESI-MS positive mode (m/z): calcd for $\mathrm{C}_{48} \mathrm{H}_{38} \mathrm{~N}_{3} \mathrm{O}_{9}\left([\mathrm{M}+\mathrm{H}]^{+}\right)$: 800.261. Found: 800.26. Anal. Calcd for $\mathrm{C}_{48} \mathrm{H}_{37} \mathrm{~N}_{3} \mathrm{O}_{9}$ : C, 72.08; H, 4.66; N, 5.25. Found: C, 70.22; H, 4.73; N, 5.24.

\section{5-(2',3',4',6'-Tetra- $O$-benzoyl- $\beta$-D-glucopyranosyl)-1-(2-hydroxyethyl)-3-phenyl-1,2,4- triazole (45)}

Prepared from compound $19(0.10 \mathrm{~g}, 0.13 \mathrm{mmol})$ and 2-hydroxyethylhydrazine $(10 \mu \mathrm{L}, 0.15$ mmol) according to general procedure IV. Purified by column chromatography (1:2 EtOAc/hexane) to give $0.09 \mathrm{~g}(90 \%)$ yellow amorphous solid. $\mathrm{R}_{\mathrm{f}}=0.45(1: 1 \mathrm{EtOAc} / \mathrm{hexane})$; $\left.[\alpha]_{\mathrm{D}}=+4\left(\mathrm{c} 0.305, \mathrm{CHCl}_{3}\right) ;{ }^{1} \mathrm{H} \mathrm{NMR}\left(360 \mathrm{MHz}, \mathrm{CDCl}_{3}\right) \delta(\mathrm{ppm}):\right): 8.09(2 \mathrm{H}, \mathrm{dd}, J=8.4,1.4$ Hz, aromatics), 7.97 (2H, dd, $J=8.3,1.2 \mathrm{~Hz}$, aromatics), $7.89(2 \mathrm{H}, \mathrm{dd}, J=8.3,1.2 \mathrm{~Hz}$, aromatics), $7.80(2 \mathrm{H}, \mathrm{dd}, J=8.4,1.4 \mathrm{~Hz}$, aromatics), $7.74(2 \mathrm{H}, \mathrm{dd}, J=7.6,2.1 \mathrm{~Hz}$, 
aromatics), 7.60-7.24 (15H, m, aromatics), 6.11 (pseudo t, $1 \mathrm{H}, J=9.6 \mathrm{~Hz}, \mathrm{H}-2$ ' or H-3' or H4'), 5.95 (1H, pseudo t, $J=9.7 \mathrm{~Hz}, \mathrm{H}-2$ ' or $\mathrm{H}-3$ ' or H-4'), $5.87(1 \mathrm{H}$, pseudo t, $J=9.8 \mathrm{~Hz}, \mathrm{H}-$ 2' or H-3' or H-4'), 5.27 (1H, d, $J=9.9 \mathrm{~Hz}, \mathrm{H}-1$ '), 4.79 (1H, dd, $J=12.4,2.7 \mathrm{~Hz}, \mathrm{H}-6$ 'a), $4.65\left(1 \mathrm{H}, \mathrm{ddd}, J=14.2,7.6,3.1 \mathrm{~Hz}, \mathrm{CH}_{2}\right), 4.51(1 \mathrm{H}, \mathrm{dd}, J=12.4,4.7 \mathrm{~Hz}, \mathrm{H}-6$ 'b), 4.42-4.29 $\left(2 \mathrm{H}, \mathrm{m}, \mathrm{H}-5^{\prime}\right.$ and $\left.\mathrm{CH}_{2}\right), 4.11-4.03\left(1 \mathrm{H}, \mathrm{m}, \mathrm{CH}_{2}\right), 3.92\left(\mathrm{ddd}, J=12.3,5.7,3.1 \mathrm{~Hz}, 1 \mathrm{H}, \mathrm{CH}_{2}\right)$, 3.52 (br s, $1 \mathrm{H}, \mathrm{OH}) ;{ }^{13} \mathrm{C} \mathrm{NMR}\left(90 \mathrm{MHz}, \mathrm{CDCl}_{3}\right) \delta(\mathrm{ppm}): 166.1,165.8,165.5,165.2(\mathrm{C}=\mathrm{O})$, 161.3, 150.8 (triazole C-3, C-5), 133.6-126.2 (aromatics), 77.2, 73.9, 73.5, 71.5, 71.4, 68.9 (C-1'-C-5', $\left.\mathrm{CH}_{2}\right), 64.8\left(\mathrm{C}^{\prime} 6^{\prime}\right), 61.4\left(\mathrm{CH}_{2}\right)$. ESI-MS positive mode (m/z): calcd for $\mathrm{C}_{44} \mathrm{H}_{38} \mathrm{~N}_{3} \mathrm{O}_{10}\left([\mathrm{M}+\mathrm{H}]^{+}\right)$: 768.256. Found: 768.28. Anal. Calcd for $\mathrm{C}_{44} \mathrm{H}_{37} \mathrm{~N}_{3} \mathrm{O}_{10}: \mathrm{C}, 68.83 ; \mathrm{H}$, 4.86; N, 5.47. Found: C, 68.96; H, 4.99; N, 5.60.

\section{5-(2',3',4',6'-Tetra- $O$-benzoyl- $\beta$-D-glucopyranosyl)-1-(tert-butyl)-3-phenyl-1,2,4-triazole}

\section{(46)}

Prepared from compound $19(0.15 \mathrm{~g}, 0.20 \mathrm{mmol})$ and tert-butylhydrazine hydrochloride $(0.03$ g, $0.24 \mathrm{mmol})$ according to general procedure IV. Purified by column chromatography $(1: 2$ EtOAc/hexane) to give $0.09 \mathrm{~g}(60 \%)$ yellow amorphous solid. $\mathrm{R}_{\mathrm{f}}=0.57$ (1:2 EtOAc/hexane); $[\alpha]_{\mathrm{D}}=+12\left(\mathrm{c} 0.485, \mathrm{CHCl}_{3}\right) ;{ }^{1} \mathrm{H} \mathrm{NMR}\left(360 \mathrm{MHz}, \mathrm{CDCl}_{3}\right) \delta(\mathrm{ppm}): 8.04-7.87(8 \mathrm{H}, \mathrm{m}$, aromatics), $7.75(2 \mathrm{H}, \mathrm{d}, J=7.4 \mathrm{~Hz}$, aromatics), 7.58-7.22 (15H, m, aromatics), 6.59 (pseudo t, $1 \mathrm{H}, J=9.6 \mathrm{~Hz}, \mathrm{H}-2^{\prime}$ ' or H-3' or H-4'), $6.04(1 \mathrm{H}$, pseudo t, $J=9.6 \mathrm{~Hz}, \mathrm{H}-2$ ' or H-3' or H4'), 5.74 (1H, pseudo t, $J=9.7 \mathrm{~Hz}, \mathrm{H}-2^{\prime}$ or $\mathrm{H}-3^{\prime}$ or $\mathrm{H}-4$ ') $5.25(1 \mathrm{H}, \mathrm{d}, J=9.7 \mathrm{~Hz}, \mathrm{H}-1$ '), 4.65 (1H, dd, $J=12.3,2.5 \mathrm{~Hz}, \mathrm{H}-6$ 'a), 4.49 (1H, dd, $J=12.2,6.9 \mathrm{~Hz}, \mathrm{H}-6$ 'b), 4.38 (1H, ddd, $J=$ 9.8, 6.9, 2.6 Hz, H-5'), $1.71\left(9 \mathrm{H}, \mathrm{s}, \mathrm{C}\left(\mathrm{CH}_{3}\right)_{3}\right) ;{ }^{13} \mathrm{C} \mathrm{NMR}\left(90 \mathrm{MHz}, \mathrm{CDCl}_{3}\right) \delta(\mathrm{ppm}): 166.2$, 166.2, 165.4, 164.6 (C=O), 159.0, 149.8 (triazole C-3, C-5), 133.7-126.3 (aromatics), 76.9, 74.6, 72.6, 71.1, 69.8 (C-1'-C-5'), 63.9 (C-6'), $61.2\left(C\left(\mathrm{CH}_{3}\right)_{3}\right), 30.3\left(\mathrm{C}\left(\mathrm{CH}_{3}\right)_{3}\right)$. ESI-MS positive mode $(\mathrm{m} / \mathrm{z})$ : calcd for $\mathrm{C}_{46} \mathrm{H}_{42} \mathrm{~N}_{3} \mathrm{O}_{9}\left([\mathrm{M}+\mathrm{H}]^{+}\right)$: 780.292. Found: 780.32. Anal. Calcd 
for $\mathrm{C}_{46} \mathrm{H}_{41} \mathrm{~N}_{3} \mathrm{O}_{9}$ : C, 70.85, H, 5.30; N, 5.39. Found: C, 70.97; H, 5.41; N, 5.34.

\section{5-(2',3',4',6'-Tetra-O-benzoyl- $\beta$-D-glucopyranosyl)-1-(3-chlorophenyl)-3-phenyl-1,2,4-}

\section{triazole (47)}

Prepared from compound $19(0.15 \mathrm{~g}, 0.20 \mathrm{mmol})$ and 3-chlorophenylhydrazine hydrochloride $(0.04 \mathrm{~g}, 0.24 \mathrm{mmol})$ according to general procedure IV. Purified by column chromatography (1:3 EtOAc/hexane) to give $0.12 \mathrm{~g}(72 \%)$ yellow amorphous solid. $\mathrm{R}_{\mathrm{f}}=0.50(1: 2$ EtOAc/hexane); $[\alpha]_{\mathrm{D}}=+27\left(\mathrm{c} 0.425, \mathrm{CHCl}_{3}\right) ;{ }^{1} \mathrm{H} \mathrm{NMR}\left(360 \mathrm{MHz}, \mathrm{CDCl}_{3}\right) \delta(\mathrm{ppm}): 8.10$ $8.00(4 \mathrm{H}, \mathrm{m}$, aromatics), $7.93(2 \mathrm{H}, \mathrm{d}, J=7.4 \mathrm{~Hz}$, aromatics $), 7.86(2 \mathrm{H}, \mathrm{d}, J=7.4 \mathrm{~Hz}$, aromatics), 7.77-7.65 (3H, m, aromatics), 7.60-7.21 (18H, m, aromatics), 6.39 (pseudo t, 1H, $J=9.7 \mathrm{~Hz}, \mathrm{H}-2^{\prime}$ ' or H-3' or H-4'), 5.97 (1H, pseudo t, $J=9.6 \mathrm{~Hz}, \mathrm{H}-2$ ' or H-3' or H-4'), 5.76 (1H, pseudo t, $J=9.7 \mathrm{~Hz}, \mathrm{H}-2^{\prime}$ or $\mathrm{H}-3$ ' or H-4'), $5.01\left(1 \mathrm{H}, \mathrm{d}, J=9.8 \mathrm{~Hz}, \mathrm{H}-1^{\prime}\right), 4.72(1 \mathrm{H}, \mathrm{dd}$, $J=12.4,2.6 \mathrm{~Hz}, \mathrm{H}-6$ 'a), 4.46 (1H, dd, $J=12.3,6.3 \mathrm{~Hz}, \mathrm{H}-6$ 'b), 4.33 (1H, ddd, $J=9.4,6.3$, $2.7 \mathrm{~Hz}, \mathrm{H}-5$ '); ${ }^{13} \mathrm{C} \mathrm{NMR}\left(90 \mathrm{MHz}, \mathrm{CDCl}_{3}\right) \delta(\mathrm{ppm}): 166.2,166.0,165.3,164.6(\mathrm{C}=\mathrm{O}), 162.3$, 150.7 (triazole C-3, C-5), 138.1-122.8 (aromatics), 77.1, 74.2, 71.8, 70.7, 69.5 (C-1'-C-5'), $63.5\left(\mathrm{C}-6\right.$ '). ESI-MS positive mode (m/z): calcd for $\mathrm{C}_{48} \mathrm{H}_{37} \mathrm{ClN}_{3} \mathrm{O}_{9}\left([\mathrm{M}+\mathrm{H}]^{+}\right)$: 834.222.

Found: 834.26. Anal. Calcd for $\mathrm{C}_{48} \mathrm{H}_{36} \mathrm{ClN}_{3} \mathrm{O}_{9}$ : C, 69.11; H, 4.35; N, 5.04. Found: C, 69.26; $\mathrm{H}, 4.32 ; \mathrm{N}, 5.15$.

\section{5-( $\beta$-D-Glucopyranosyl)-3-methyl-1-phenyl-1,2,4-triazole (48)}

From triazole $42(0.10 \mathrm{~g}, 0.14 \mathrm{mmol})$ according to general procedure V. Purified by column chromatography $\left(18: 1 \mathrm{CHCl}_{3} / \mathrm{MeOH}\right)$ to yield $0.04 \mathrm{~g}(96 \%)$ white amorphous solid. $\mathrm{R}_{\mathrm{f}}=0.27$ $\left(9: 1 \mathrm{CHCl}_{3} / \mathrm{MeOH}\right) ;[\alpha]_{\mathrm{D}}=+7(\mathrm{c} 0.31, \mathrm{MeOH}) ; 1 \mathrm{H} \mathrm{NMR}\left(360 \mathrm{MHz}, \mathrm{CD}_{3} \mathrm{OD}\right) \delta(\mathrm{ppm}): 7.61-$ $7.50(5 \mathrm{H}, \mathrm{m}$, aromatics $), 4.30(1 \mathrm{H}, \mathrm{d}, J=9.6 \mathrm{~Hz}, \mathrm{H}-1$ ' $), 3.91-3.82(2 \mathrm{H}, \mathrm{m}, \mathrm{H}-2$ ' or H-3' or H4' and H-6'a), 3.67 (1H, dd, $J=12.1,5.2 \mathrm{~Hz}, \mathrm{H}-6$ 'b), 3.49 (1H, pseudo t, $J=9.3 \mathrm{~Hz}, \mathrm{H}-2$ ' or 
H-3' or H-4'), 3.40 (1H, pseudo t, $J=9.0 \mathrm{~Hz}, \mathrm{H}-2^{\prime}$ or H-3' or H-4'), 3.31-3.28 (1H, m, H-5'), $2.42\left(3 \mathrm{H}, \mathrm{s}, \mathrm{CH}_{3}\right) ;{ }^{13} \mathrm{C} \mathrm{NMR}\left(90 \mathrm{MHz}, \mathrm{CD}_{3} \mathrm{OD}\right) \delta(\mathrm{ppm}): 160.9,154.1$ (triazole C-3, C-5), 137.2 (q, Ph), 130.1, 130.1, 126.0 (aromatics), 81.5, 78.6, 73.4, 72.8, 70.3 (C-1'-C-5'), 62.2 (C-6'), $13.6\left(\mathrm{CH}_{3}\right)$. ESI-MS positive mode $(\mathrm{m} / \mathrm{z})$ : calcd for $\mathrm{C}_{15} \mathrm{H}_{19} \mathrm{~N}_{3} \mathrm{NaO}_{5}\left([\mathrm{M}+\mathrm{Na}]^{+}\right)$: 344.122. Found: 344.12. Anal. Calcd for $\mathrm{C}_{15} \mathrm{H}_{19} \mathrm{~N}_{3} \mathrm{O}_{5}$ : C, 56.07; H, 5.96; N, 13.08. Found: C, 56.20; H, 6.03; N, 13.01.

\section{5-( $\beta$-D-Glucopyranosyl)-1,3-diphenyl-1,2,4-triazole (49)}

From triazole $44(0.14 \mathrm{~g}, 0.18 \mathrm{mmol})$ according to general procedure V. Purified by column chromatography $\left(18: 1 \mathrm{CHCl}_{3} / \mathrm{MeOH}\right)$ to yield $0.05 \mathrm{~g}(83 \%)$ white amorphous solid. $\mathrm{R}_{\mathrm{f}}=0.28$ $\left(9: 1 \mathrm{CHCl}_{3} / \mathrm{MeOH}\right) ;[\alpha]_{\mathrm{D}}=+7(\mathrm{c} 0.47, \mathrm{MeOH}) ; 1 \mathrm{H} \mathrm{NMR}\left(360 \mathrm{MHz}, \mathrm{CD}_{3} \mathrm{OD}\right) \delta(\mathrm{ppm})$ : 8.09-8.06 (2H, m, aromatics), 7.70-7.67 (2H, m, aromatics), 7.55-7.50 (3H, m, aromatics), 7.45-7.39 (3H, m, aromatics), $4.40\left(1 \mathrm{H}, \mathrm{m}, \mathrm{H}-1^{\prime}\right), 4.00(1 \mathrm{H}$, pseudo t, $J=9.3 \mathrm{~Hz}, \mathrm{H}-2$ ' or H3' or H-4'), 3.81 (1H, dd, $J=12.1,2.5 \mathrm{~Hz}, \mathrm{H}-6$ 'a), 3.71 (1H, dd, $J=12.4,4.4 \mathrm{~Hz}, \mathrm{H}-6$ 'b), 3.61 (1H, pseudo t, $J=9.3 \mathrm{~Hz}, \mathrm{H}-2^{\prime}$ or H-3' or H-4'), $3.45\left(1 \mathrm{H}\right.$, pseudo t, $J=9.1 \mathrm{~Hz}, \mathrm{H}-2^{\prime}$ ' or H-3' or H-4'), 3.32 (1H, ddd, $J=9.7,4.4,2.5 \mathrm{~Hz}, \mathrm{H}-5$ ' $) ;{ }^{13} \mathrm{C} \mathrm{NMR}\left(90 \mathrm{MHz}, \mathrm{CD}_{3} \mathrm{OD}\right) \delta$ (ppm): 162.1, 154.5 (triazole C-3, C-5), 137.1, 130.1 (q, Ph), 130.0, 129.8, 129.0, 126.9, 125.9 (aromatics), 80.9, 78.1, 73.2, 72.7, 69.7 (C-1'-C-5'), 61.7 (C-6'). ESI-MS positive mode (m/z): calcd for $\mathrm{C}_{20} \mathrm{H}_{21} \mathrm{~N}_{3} \mathrm{NaO}_{5}\left([\mathrm{M}+\mathrm{Na}]^{+}\right)$: 406.138. Found: 406.17. Anal. Calcd for $\mathrm{C}_{20} \mathrm{H}_{21} \mathrm{~N}_{3} \mathrm{O}_{5}$ : C, 62.65; H, 5.52; N, 10.96. Found: C, 62.80; H, 5.48; N, 11.02.

\section{5-( $\beta$-D-Glucopyranosyl)-1-(2-hydroxyethyl)-3-phenyl-1,2,4-triazole (50)}

From triazole 45 ( $0.09 \mathrm{~g}, 0.14 \mathrm{mmol})$ according to general procedure V. Purified by column chromatography $\left(9: 1 \mathrm{CHCl}_{3} / \mathrm{MeOH}\right)$ to yield $0.04 \mathrm{~g}(98 \%)$ white amorphous solid. $\mathrm{R}_{\mathrm{f}}=0.10$ $\left(9: 1 \mathrm{CHCl}_{3} / \mathrm{MeOH}\right) ;[\alpha]_{\mathrm{D}}=+1(\mathrm{c} 0.35, \mathrm{MeOH}) ; 1 \mathrm{H} \mathrm{NMR}\left(360 \mathrm{MHz}, \mathrm{CD}_{3} \mathrm{OD}\right) \delta(\mathrm{ppm}): 8.01$ 
(2H, d, $J=6.5 \mathrm{~Hz}$, aromatics), 7.43-7.38 (3H, m, aromatics), 4.67 (1H, d, $J=9.6 \mathrm{~Hz}, \mathrm{H}-1$ ') 4.49-4.33 (2H, m, H-2' and/or H-3' and/or H-4'), 4.00-3.95 (2H, m, $\left.\mathrm{CH}_{2}\right), 3.88(1 \mathrm{H}, \mathrm{dd}, J=$ 12.2, $1.9 \mathrm{~Hz}, \mathrm{H}-6$ 'a), 3.79 (1H, t, $J=9.5 \mathrm{~Hz}, \mathrm{H}-2^{\prime}$ ' or H-3' or H-4'), 3.73 (1H, dd, $J=12.2$, $4.8 \mathrm{~Hz}, \mathrm{H}-6$ 'b), 3.58-3.52 (2H, m, $\left.\mathrm{CH}_{2}\right), 3.50-3.44$ (1H, m, H-5'); ${ }^{13} \mathrm{C} \mathrm{NMR}(90 \mathrm{MHz}$, $\left.\mathrm{CD}_{3} \mathrm{OD}\right) \delta(\mathrm{ppm}): 161.9,155.9$ (triazole C-3, C-5), 130.5 (q, Ph), 130.2, 129.4, 127.1 (aromatics), 81.8, 78.8, 74.2, 73.8, 70.5 (C-1'-C-5'), 62.3 (C-6'), 61.3, 52.2 (2 × $\left.\mathrm{CH}_{2}\right)$. ESIMS positive mode (m/z): calcd for $\mathrm{C}_{16} \mathrm{H}_{21} \mathrm{~N}_{3} \mathrm{NaO}_{6}\left([\mathrm{M}+\mathrm{Na}]^{+}\right)$: 374.133. Found: 374.13 . Anal. Calcd for $\mathrm{C}_{16} \mathrm{H}_{21} \mathrm{~N}_{3} \mathrm{O}_{6}$ : C, 54.70; H, 6.02; N, 11.96. Found: C, 54.85; H, 6.00; N, 12.04 .

\section{1-(tert-Butyl)-5-( $\beta$-D-glucopyranosyl)-3-phenyl-1,2,4-triazole (51)}

From triazole 46 ( $0.09 \mathrm{~g}, 0.14 \mathrm{mmol})$ according to general procedure V. Purified by column chromatography $\left(18: 1 \mathrm{CHCl}_{3} / \mathrm{MeOH}\right)$ to yield $0.04 \mathrm{~g}(99 \%)$ white amorphous solid. $\mathrm{R}_{\mathrm{f}}=0.23$ $\left(9: 1 \mathrm{CHCl}_{3} / \mathrm{MeOH}\right) ;[\alpha]_{\mathrm{D}}=+15(\mathrm{c} 0.295, \mathrm{MeOH}) ; 1 \mathrm{H} \mathrm{NMR}\left(360 \mathrm{MHz}, \mathrm{CD}_{3} \mathrm{OD}\right) \delta(\mathrm{ppm})$ : $7.99(2 \mathrm{H}, \mathrm{d}, J=6.7 \mathrm{~Hz}$, aromatics), 7.40-7.34 (3H, m, aromatics), $4.66(1 \mathrm{H}, \mathrm{d}, J=9.3 \mathrm{~Hz}, \mathrm{H}-$ 1'), $4.08(1 \mathrm{H}$, pseudo t, $J=9.0 \mathrm{~Hz}, \mathrm{H}-2$ ' or H-3' or H-4'), $3.83(1 \mathrm{H}, \mathrm{dd}, J=12.2,2.5 \mathrm{~Hz}, \mathrm{H}-$ 6'a), 3.75 (1H, dd, $J=12.3,3.9$ Hz, H-6'b), 3.68-3.54 (2H, m, H-2' and/or H-3' and/or H-4'), 3.43-3.38 (1H, m, H-5'), $1.72\left(9 \mathrm{H}, \mathrm{s}, \mathrm{C}\left(\mathrm{CH}_{3}\right)_{3}\right) ;{ }^{13} \mathrm{C} \mathrm{NMR}\left(90 \mathrm{MHz}, \mathrm{CD}_{3} \mathrm{OD}\right) \delta(\mathrm{ppm})$ : 159.1, 153.3 (triazole C-3, C-5), 131.2 (q, Ph), 129.4, 128.8, 126.5, (aromatics), 80.7, 77.9, 74.0, 73.2, $69.7\left(\mathrm{C}-1^{\prime}-\mathrm{C}-5\right.$ ' $), 62.0\left(\mathrm{C}^{\prime}{ }^{\prime}\right.$ '), $61.7\left(\mathrm{C}\left(\mathrm{CH}_{3}\right)_{3}\right), 30.5\left(\mathrm{C}\left(\mathrm{CH}_{3}\right)_{3}\right)$. ESI-MS positive mode $(\mathrm{m} / \mathrm{z})$ : calcd for $\mathrm{C}_{18} \mathrm{H}_{25} \mathrm{~N}_{3} \mathrm{NaO}_{5}\left([\mathrm{M}+\mathrm{Na}]^{+}\right)$: 386.170. Found: 386.17. Anal. Calcd for $\mathrm{C}_{18} \mathrm{H}_{25} \mathrm{~N}_{3} \mathrm{O}_{5}: \mathrm{C}, 59.49 ; \mathrm{H}, 6.93 ; \mathrm{N}, 11.56$. Found: C, 59.56; H, 6.90; N, 11.63.

\section{1-(3-Chlorophenyl)-5-( $\beta$-D-glucopyranosyl)-3-phenyl-1,2,4-triazole (52)}

From triazole 47 ( $0.12 \mathrm{~g}, 0.14 \mathrm{mmol})$ according to general procedure V. Purified by column chromatography $\left(18: 1 \mathrm{CHCl}_{3} / \mathrm{MeOH}\right)$ to yield $0.06 \mathrm{~g}(99 \%)$ white amorphous solid. $\mathrm{R}_{\mathrm{f}}=0.26$ 
$\left(9: 1 \mathrm{CHCl}_{3} / \mathrm{MeOH}\right) ;[\alpha]_{\mathrm{D}}=+9(\mathrm{c} 0.5, \mathrm{MeOH}) ; 1 \mathrm{H} \mathrm{NMR}\left(360 \mathrm{MHz}, \mathrm{CD}_{3} \mathrm{OD}\right) \delta(\mathrm{ppm}): 8.11-$ $8.03(2 \mathrm{H}, \mathrm{m}$, aromatics), $7.76(1 \mathrm{H}, \mathrm{s}$, aromatics $), 7.68-7.63(1 \mathrm{H}, \mathrm{m}$, aromatics $), 7.50-7.38$ (5H, m, aromatics), $4.39\left(1 \mathrm{H}, \mathrm{d}, J=9.6 \mathrm{~Hz}, \mathrm{H}-1^{\prime}\right), 4.02(1 \mathrm{H}$, pseudo t, $J=9.3 \mathrm{~Hz}, \mathrm{H}-2$ ' or H3' or H-4'), 3.85 (1H, dd, $J=12.2,2.5 \mathrm{~Hz}, \mathrm{H}-6$ 'a), 3.74 (1H, dd, $J=12.3,3.9 \mathrm{~Hz}, \mathrm{H}-6$ 'b), $3.61\left(1 \mathrm{H}\right.$, pseudo t, $J=9.3 \mathrm{~Hz}, \mathrm{H}-2^{\prime}$ or $\mathrm{H}-3^{\prime}$ ' or H-4'), $3.48(1 \mathrm{H}$, pseudo t, $J=9.3 \mathrm{~Hz}, \mathrm{H}-2$ ' or H-3' or H-4'), 3.36 (1H, ddd, $J=9.8,4.5,2.5 \mathrm{~Hz}, \mathrm{H}-5$ ' $) ;{ }^{13} \mathrm{C} \mathrm{NMR}\left(90 \mathrm{MHz}, \mathrm{CD}_{3} \mathrm{OD}\right) \delta$ (ppm): 162.4, 154.6 (triazole C-3, C-5), 138.2, 135.5 (q, Ph), 131.0 130.3, 130.3, 130.0, 129.1, 126.9, 126.1, 124.1 (aromatics), 81.1, 78.2, 73.1, 72.8, 69.7 (C-1'-C-5'), 61.7 (C-6'). ESI-MS positive mode (m/z): calcd for $\mathrm{C}_{20} \mathrm{H}_{20} \mathrm{ClN}_{3} \mathrm{NaO}_{5}\left([\mathrm{M}+\mathrm{Na}]^{+}\right)$: 440.099. Found: 440.10. Anal. Calcd for $\mathrm{C}_{20} \mathrm{H}_{20} \mathrm{ClN}_{3} \mathrm{O}_{5}$ : C, 57.49; H, 4.82; N, 10.06. Found: C, 57.60; H, 4.80; N, 10.11 .

\section{Supporting information}

Copies of NMR spectra for the new compounds.

\section{Acknowledgement}

This work was supported by the Hungarian Scientific Research Fund (OTKA 109450). T. Docsa is thanked for the enzyme assays. 


\section{References}

1. Potts, K. T. Chem. Rev. 1961, 61, 87-127.

2. Polya, J. B. In Comprehensive Heterocyclic Chemistry; Potts, K. T. Ed.; Pergamon: Exeter, 1984; pp. 733-790.

3. Garratt, P. J. In Comprehensive Heterocyclic Chemistry II; Katritzky, A. R.; Rees, C. W.; , E. F. V. S. Eds.; Elsevier, 1996; pp. 127-163.

4. Al-Masoudi, I. A.; Al-Souda, Y. A.; Al-Salihi, N. J.; Al-Masoudi, N. A. Chem. Heterocycl. Comp. 2006, 42, 1377-1403.

5. Curtis, A. D. M.; Jennings. In Comprehensive Heterocyclic Chemistry III; Katritzky, A. R.; Ramsden, C. A.; Scriven, E. F. V.; Taylor, R. J. K. Eds.; Elsevier, 2008; pp. 159-209.

6. Maddila, S.; Pagadala, R.; Jonnalagadda, S. B. Lett. Org. Chem. 2013, 10, 693-714.

7. Zhang, H.-Z.; Damu, G. L. V.; Cai, G.-X.; Zhou, C.-H. Curr. Org. Chem. 2014, 18, $359-406$.

8. Shneine, J. K.; Alaraji, Y. H. Int. J. Sci. Res. 2016, 5, 1411-1423.

9. Zeidler, J.; Baraniak, D.; Ostrowski, T. Eur. J. Med. Chem. 2015, 97, 409-418.

10. Al-Masoudi, N. A.; Issa, F. B.; Al-Timari, U. A. Bull. Soc. Chim. Belg. 1997, 106, 215-220.

11. El Ashry, E. S. H.; Awad, L. F.; Winkler, M. Perkin 1 2000, 829-834.

12. Yu, J.; Li, Z.; Lu, W.; Zhang, S.; Cai, M. Tetrahedron: Asymm. 2003, 14, 2507-2513.

13. Libnow, S.; Wille, S.; Christiansen, A.; Hein, M.; Reinke, H.; Koeckerling, M.; Miethchen, R. Synthesis 2006, 496-508.

14. El Ashry, E. S. H.; Kassem, A. A.; Abdel-Hamid, H. M.; Louis, F.; Khattab, S. A. N.; Aouad, M. R. Carbohydr. Res. 2009, 344, 725-733.

15. Shen, G. Y.; Robins, R. K.; Revankar, G. R. Nucl. Nucl. 1991, 10, 1707-1717. 
16. Al-Masoudi, N.; Hassan, N. A.; Al-Soud, Y. A.; Schmidt, P.; Gaafar, A.; Weng, M.; Marino, S.; Schoch, A.; Amer, A.; Jochims, J. C. J. Chem. Soc. Perkin. Trans. 1 1998, 947-953.

17. Al-Masoudi, N. A.; Al-Soud, Y. A.; Ali, I. A. I. Nucl. Nucl. Nucl. Acids 2007, 26, 3743.

18. Bokor, É.; Kun, S.; Goyard, D.; Tóth, M.; Praly, J.-P.; Vidal, S.; Somsák, L. Chem. Rev. 2017, 117, 1687-1764.

19. Bokor, É.; Docsa, T.; Gergely, P.; Somsák, L. ACS Med. Chem. Lett. 2013, 4, 612615.

20. Bokor, É.; Fekete, A.; Varga, G.; Szőcs, B.; Czifrák, K.; Komáromi, I.; Somsák, L. Tetrahedron 2013, 69, 10391-10404.

21. Kun, S.; Bokor, É.; Varga, G.; Szőcs, B.; Páhi, A.; Czifrák, K.; Tóth, M.; Juhász, L.; Docsa, T.; Gergely, P.; Somsák, L. Eur. J. Med. Chem. 2014, 76, 567-579.

22. Somsák, L.; Bokor, É.; Czibere, B.; Czifrák, K.; Koppány, C.; Kulcsár, L.; Kun, S.; Szilágyi, E.; Tóth, M.; Docsa, T.; Gergely, P. Carbohydr. Res. 2014, 399, 38-48.

23. Szőcs, B.; Bokor, É.; Szabó, K. E.; Kiss-Szikszai, A.; Tóth, M.; Somsák, L. RSC Adv. 2015, 5, 43620-43629.

24. Bokor, É.; Széles, Z.; Docsa, T.; Gergely, P.; Somsák, L. Carbohydr. Res. 2016, 429, 128-134.

25. Somsák, L.; Nagy, V. Tetrahedron: Asymm. 2000, 11, 1719-1727. Corrigendum 2247.

26. Prachayasittikul, S.; Bauer, L. J. Het. Chem. 1985, 22, 771-775.

27. Inamoto, K.; Saito, T.; Hiroya, K.; Doi, T. J. Org. Chem. 2010, 75, 3900-3903.

28. Black, D. S.; Rothnie, N. E. Aust. J. Chem. 1983, 36, 1141-1147.

29. Sibi, M. P.; Prabagaran, N.; Ghorpade, S. G.; Jasperse, C. P. J. Am. Chem. Soc. 2003, $125,11796-11797$. 
30. Baburao, K.; Costello, A. M.; Petterson, R. C.; Sander, G. E. J. Chem. Soc. C 1968, 2779-81.

31. Thompson, Q. E. J. Am. Chem. Soc. 1951, 73, 5841-6.

32. Ösz, E.; Czifrák, K.; Deim, T.; Szilágyi, L.; Bényei, A.; Somsák, L. Tetrahedron 2001, 57, 5429-5434.

33. Goerdeler, J.; Horstmann, H. Chem. Ber. 1960, 93, 663-670.

34. Czifrák, K.; Szilágyi, P.; Somsák, L. Tetrahedron: Asymm. 2005, 16, 127-141.

35. Somsák, L.; Kovács, L.; Tóth, M.; Ösz, E.; Szilágyi, L.; Györgydeák, Z.; Dinya, Z.; Docsa, T.; Tóth, B.; Gergely, P. J. Med. Chem. 2001, 44, 2843-2848. 